\title{
Poking microtubules bring about nuclear wriggling to position nuclei
}

\author{
Ph.D. Thesis
}

Szilárd Szikora

\author{
Department of Biology \\ Faculty of Medicine \\ University of Szeged \\ and the
}

Biochemistry, Biophysics and Cell Biology

Ph.D. Program

Supervisor: Professor János Szabad

Szeged, 2012 


\section{LIST OF CONTENTS}

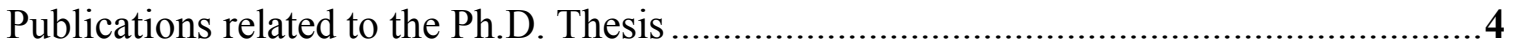

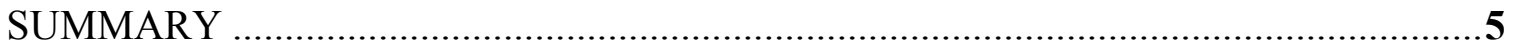

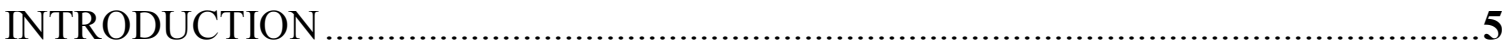

MATERIALS AND METHODS _.......................................................................

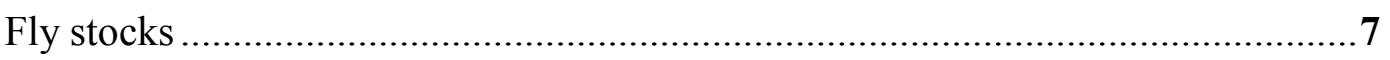

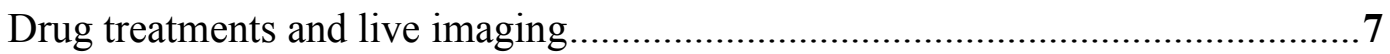

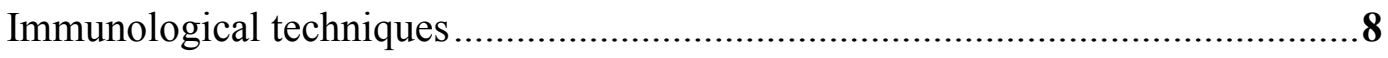

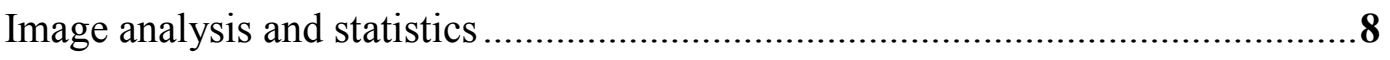

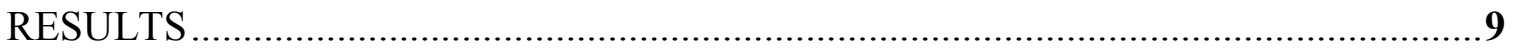

Importin- $\beta$-GFP speckles wriggle in the nuclear envelope of the Drosophila

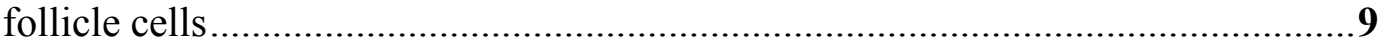

The speckles moving about are indicators of the wriggling of the entire nucleus...9

Features of nuclear wriggling ................................................................10

Nuclei drift along the apical-basal axis.........................................................11

In the absence of the actin cytoskeleton, nuclear wriggling speeds up and the nuclei become mispositioned ........................................................................ 13

MT motor functions do not seem to be required for nuclear wriggling .................15

Dynamic MTs render the nuclei wriggling ......................................................16

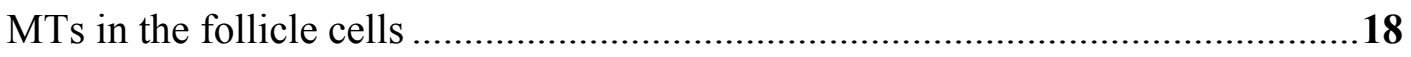

Dynamic MTs exert force on the nuclei …….....................................................19

Growing MTs tuck in the nuclear envelope .................................................21

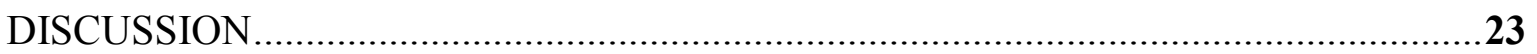

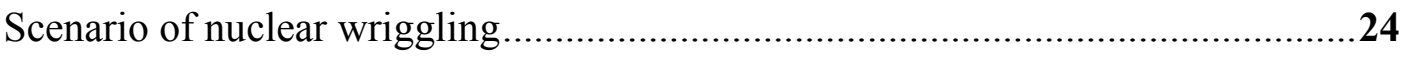

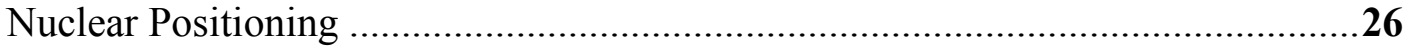

The nuclear positioning machineries ............................................................27

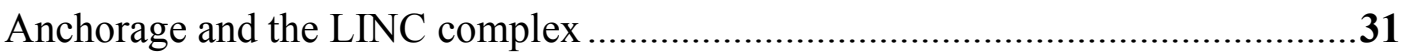

Defective nuclear positioning and diseases ...................................................33

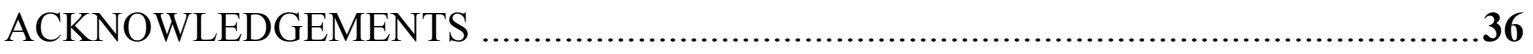

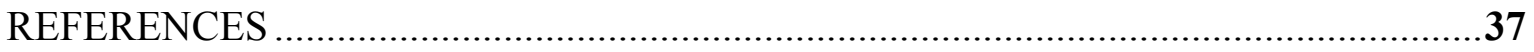

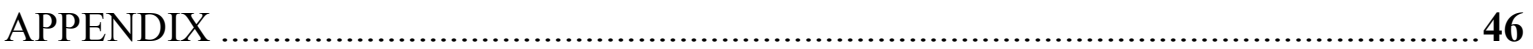




\section{Publications related to the Ph.D. Thesis}

\section{Scientific publications}

Szilárd Szikora, Imre Gáspár and János Szabad, Poking microtubules bring about nuclear wriggling to position nuclei.

Journal Cell Science, accepted for publication (September 17, 2012). IF: 6.110

\section{Related papers}

Zoltán Villányi, Bernadett Papp, Szilárd Szikora, Imre Boros, and János Szabad, The DRE motif is a key component in the expression regulation of the importin- $\beta$ encoding Ketel gene in Drosophila.

Mechanisms of Development 125, 822-831, 2008. IF: 3.160

Zoltán Villányi, Imre Gáspár, Szilárd Szikora, László G. Puskás and János Szabad, Importin- $\beta$ and peroxiredoxin-6005 are involved in mitochondrial biogenesis.

Mechanisms of Development 128, 191-199, 2011. IF: 3.160

\section{“Making science popular" papers}

Szikora Szilárd, Gáspár Imre és Szabad János, Hogyan kerülnek helyükre a sejtmagok?

Természet Világa 141, 303-306, 2010.

Oral presentations (The first authors presented the papers)

Szikora Szilárd, Gáspár Imre és Szabad János, Forgolódó sejtmagvak?!

"Genetikai Mühelyek Magyarországon” VII. Minikonferencia Szeged, 2008. szeptember 9.

Szikora Szilárd, Gáspár Imre és Szabad János, Forgolódó sejtmagvak.

VIII. Magyar Genetikai Kongresszus, XV. Sejt- és Fejlődésbiológiai Napok Nyíregyháza, 2009. április 17-19.

Szilárd Szikora, Imre Gáspár and János Szabad, Wiggling nuclei reveal a novel nuclear positioning mechanism.

International Ph.D. Conference, Szeged, May 17-18, 2010.

János Szabad, Szilárd Szikora and Imre Gáspár, Nuclear Positioning. Systems Biology of Development. EMBO Workshop. Ascona, Switzerland, August 16-20, 2010. 
Szikora Szilárd, Gáspár Imre és Szabad János, Egy új sejtmag pozícionálódási mechanizmus. "Genetikai Mühelyek Magyarországon” IX. Minikonferencia, Szeged, 2010. szeptember 3.

Szikora Szilárd, Gáspár Imre és Szabad János, Sejtmag pozícionálás böködő mikrotubulusokkal. IX. Magyar Genetikai Kongresszus és XVI. Sejt- és Fejlődésbiológiai Napok, Siófok, 2011. március 25-27.

\section{Posters}

Szilárd Szikora, Imre Gáspar and János Szabad, Wiggling nuclei reveal novel nuclear positioning mechanism. EMBO Conference Series (1st) on Microtubules: structure, regulation and functions, Heidelberg, Germany, June 2-5, 2010.

Szilárd Szikora, Imre Gáspar and János Szabad, Wiggling nuclei reveal novel nuclear positioning mechanism. 42. Membrán-Transzport Konferencia, Sümeg, 2012. május 15-18. 


\section{SUMMARY}

Nuclei wriggle in the cells of the follicle epithelium of the Drosophila pre-vitellogenic egg primordia. Although similar phenomena have been reported for a number of cultured cell types and some neurons in the zebra fish embryo, the mechanism and importance of the process remained unexplained. Wriggling involves the succession of sudden and random minor turns of the nuclei, about three twists in a minute with roughly $12^{\circ}$ per twist, one of which lasts typically for 14 seconds. Wriggling is brought about by the growing microtubules seeded throughout the cell cortex, which, while poking the nuclei, buckle and exert 5-40 piconewtons over about 16 seconds - as it appears - through the nuclear pore complexes. While wriggling, the nuclei drift about $5 \mu \mathrm{m}$ in a day in the immensely growing follicle cells along the apical-basal axis from the apical to the basal cell region. An over twofold excess of the microtubules nucleated in the apical cell region, as compared to those seeded in the basal cell cortex, makes the nuclei drift along the apical-basal axis. Nuclear wriggling and positioning appear to be tightly related processes: they cease simultaneously - when the nuclei become anchored by the actin cytoskeleton -, moreover, colchicine or taxol treatments eliminate both nuclear wriggling and positioning. We propose that the wriggling nuclei reveal a thus far not described nuclear positioning mechanism.

\section{INTRODUCTION}

While analyzing ooplasmic streaming and the kinetics of lipid-droplet transport in the developing Drosophila egg primordia (Gaspar and Szabad, 2009a and 2009b), we noticed that nuclei wriggle in the follicular epithelial cells during the pre-vitellogenic stages of oogenesis. They undergo a series of sudden twists and turns without any specific directionality. A similar phenomenon has been reported a number of times during the past 60 or so years for cultured cells and some cells in the developing brain of the zebra fish embryos (Pomerat, 1953; Bard et al., 1985; De Boni and Mintz, 1986; Paddock and Albrecht-Buehler, 1986 and 1988; Herbomel, 1999). However, the mechanism and significance of nuclear wriggling remained unclear. What makes the nuclei wrigglin? What is the importance of nuclear wriggling in life of the cells?

We set out to study the mechanism of nuclear wriggling in the Drosophila follicle cells by highlighting different cell components and monitoring their behavior with time-lapse confocal microscopy under both normal conditions and disrupted cytoskeleton functions. We report that the growing microtubules (MTs) - seemingly randomly - poke the nuclei and generate forces that make the nuclei wriggling. The numerous MTs emanating from the apical and the few from the basal cell cortex achieve slow drifting of the nucleus away from the vicinity of the apical toward 
the basal membrane. MTs seeded in the lateral cell cortex keep the nuclei along the apical-basal cell axis. There is a tight correlation between nuclear wriggling and positioning of the nuclei: the nuclei stop wriggling and drifting concurrently when the actin microfilaments fix the nuclei once they reach their final destination. Colchicine and taxol treatments eliminate both nuclear wriggling and positioning. Thus, it appears that nuclear wriggling is a hallmark of a formerly not described nuclear positioning mechanism. My thesis describes a novel nuclear positioning mechanism in which the growing MTs position the nuclei by bumping into them and pushing to their right site.

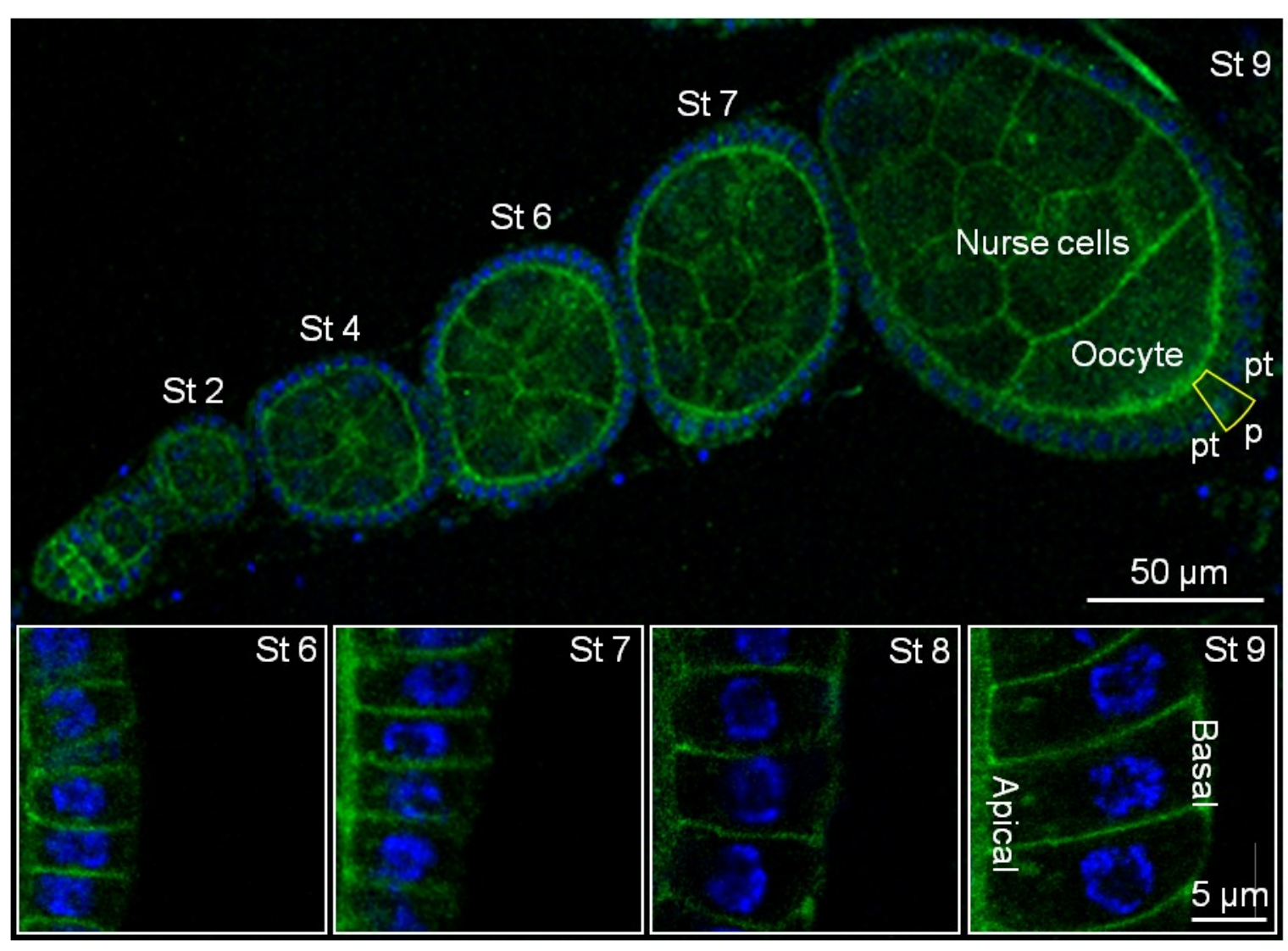

Figure 1. A Drosophila ovariole with five egg primordia in different stages (St) of development. A single layer of follicle cells envelope the oocyte and its sister nurse cells. The inserts illustrate follicle cells in the St 6 - St 9 egg primordia. While the nuclei reside near the apical membrane during St 6, they reach a final position close to the basal membrane during St 9 . The present study focused on the posterior terminal follicle cells (pt) adjacent to the polar cells ( $\mathrm{p}$, bordered in yellow). The cell membrane was highlighted by Spider-GFP and the DNA by Hoechst 33342 in the optical sections of live egg primordia. 


\section{MATERIALS AND METHODS}

\section{Fly stocks}

The Drosophila cultures were raised on standard Drosophila food and kept at $25^{\circ} \mathrm{C}$. The nuclei were outlined by highlighting either the NPCs using Importin- $\beta$-GFP (encoded by the ketel ${ }^{G F P}$ allele; Karpova et al., 2006; Villányi et al., 2008) or using lamin-GFP encoded by a UAS-laminGFP transgene (kind gift from Nico Stuurman; see the FlyBase at http://flybase.bio.indiana.edu). The chromatin was highlighted by Histone2Av-RFP (Schuh et al., 2007) and Jupiter-GFP rendered the MTs glow green (Karpova et al., 2006). The centromere was highlighted by CIDGFP (Vermaak et al., 2002), the endoplasmic reticulum by PDI-GFP (Bobinec et al., 2003; Buszczak et al., 2007) and the cell membrane by Spider-GFP (Frescas et al., 2006). The plus ends of the MTs were highlighted by driving the expression of either the UAS-EB1-GFP (Rolls et al., 2007) or the UAS-EB1-Cherry (kindly provided by Damian Brunner) transgenes. All over type of expression of the UAS-based transgenes was achieved by an Act5C-Gal4 or a Tub-Gal4 driver. We also made use of the following follicle cell-specific drivers: e22c-Gal4, slbo-Gal4 and T155-Gal4 (see the FlyBase). Dynein function was disrupted by interfering with the dynactin complex via the overexpression of human-dynamitin (Echeverri et al., 1996; Burkhardt et al., 1997). The UAS-human-dynamitin transgene was driven by e22c-Gal4. Features of the Gal4/UAS sytem were described by Brand and Perrimon (1993) and summarized in Duffy (2002). For an explanation of the genetic symbols, see the FlyBase.

\section{Drug treatments and live imaging}

To analyze the egg primordia, ovaries were dissected and the egg primordia were prepared for analysis as described earlier (Gaspar and Szabad, 2009b). Briefly, the ovarioles were dissected in BRB80 buffer ( $80 \mathrm{mM}$ Pipes, $2 \mathrm{mM} \mathrm{MgCl}_{2}$ and $1 \mathrm{mM}$ EGTA, $\mathrm{pH}=6.9$ ), incubated in BRB80 buffer for 10-30 minutes. Two types of drug treatments were applied. (1) The buffer contained one of the cytoskeletal drugs: Cytochalasin $\mathrm{D}$ to prevent actin polymerization $(25 \mu \mathrm{M}$ freshly prepared solution in $0.2 \% \mathrm{DMSO}$ ), colchicine (Sigma; $125 \mu \mathrm{M}, 30 \mathrm{~min}$ ) to depolymerize the MTs, taxol (10 nM for 10 minutes) to stabilize the MTs, lidocaine (Sigma; $50 \mathrm{mM}$ in BRB80) to block kinesin, EHNA [(erythro-9-(2-hydroxy-3-nonyl) adenine hydrochloride, Sigma; $2.5 \mathrm{mM}$ for $30 \mathrm{~min}$ ] or vanadate (Sigma; $15 \mathrm{mM}$ for $30 \mathrm{~min}$ ) to inhibit dynein. Treatment with $0.2 \%$ DMSO does not alter the behavior of the nuclei in the follicle cells. (2) One-day-old females 
were injected with $0.3 \mu 125 \mu \mathrm{M}$ Cytochalasin D or with $100 \mu \mathrm{g} / \mathrm{ml}$ colchicine solution eight hours before dissection of their ovarioles for analysis of the follicle cells, as described above.

The treated ovarioles were then transferred onto a coverslip, covered with Voltalef $10 \mathrm{~S}$ halocarbon oil after the removal of the excess buffer and analyzed in an Olympus FV1000 confocal microscope. The present analysis was restricted to the posterior terminal follicle cells, adjacent to the polar cells (Fig. 1; Horne-Badovinac and Bilder, 2005).

\section{Immunological techniques}

To analyze $\gamma$-tubulin localization in the follicle cells, ovaries were dissected in PBS from well fed wild-type females and fixed in a mixture of formaldehyde and chlorox in PBS. The solution was freshly prepared and contained $4 \%$ formaldehyde and $0.2 \%$ chlorox. To block nonspecific staining, the ovaries were incubated in 1\% BSA (Sigma) in PBST for 90 minutes at room temperature. To detect $\gamma$-tubulin, the ovaries were incubated with rabbit polyclonal antibody (Sigma T3559, 1:5000) overnight at $4^{\circ} \mathrm{C}$. After several rinses in PBST, the ovaries were incubated in secondary antibody (Alexa Fluor-633) for 3 hours at room temperature. To detect DNA, the ovaries were stained with Hoechst 33342 following the incubation in the secondary antibody. The ovaries were mounted in Aqua PolyMount (Polysciences Inc) following several rinses in PBST.

\section{Image analysis and statistics}

To analyze nuclear wriggling, we used the SpotTracker plug-in of ImageJ (http://rsb.info.nih.gov/ij/). The tracking analysis was done by a custom-made ImageJ plug-in (Secant) and an Excel macro. Images were deconvoluted using the ImageJ software. Statistical analysis of the data was carried out using SPSS 15 and the $\mathrm{P}<0.05$ differences were considered statistically significant. Linear regression lines were compared by GraphPad Prism statistical software (GraphPad Software Inc., San Diego, CA). 


\section{RESULTS}

\section{Importin- $\beta$-GFP speckles wriggle in the nuclear envelope of the Drosophila follicle cells}

We noticed during imaging developing Drosophila egg primordia that Importin- $\beta$-GFP forms a punctuate pattern over the nuclear envelope of the follicle cell nuclei (Fig. 2). Importin- $\beta$, a component of nuclear protein import, is associated with the nuclear pore complexes (NPCs; Stewart 2007; Villanyi et al 2008). Besides the regular faint spots, which denote the NPCs, a few bright speckles - most likely annulate lamellae (Delmar et al., 2008; Lau et al., 2009) - appear in the nuclear envelope (Fig. 2). As confocal time-lapse microscopy revealed, the positions of the speckles change over time rather dynamically (Fig. 2). However, their relative positions do not change, and thus the resulting punctuate-pattern remains stable over several minutes. These observations imply that the speckles do not move independently from each other, rather the underlying geometric structure undergoes continuous, rigid body transformations. As the position of the nucleus does not seem to change on the scale of the imaging, the above transformations are best described as wriggling of the object.

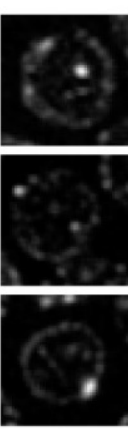

0
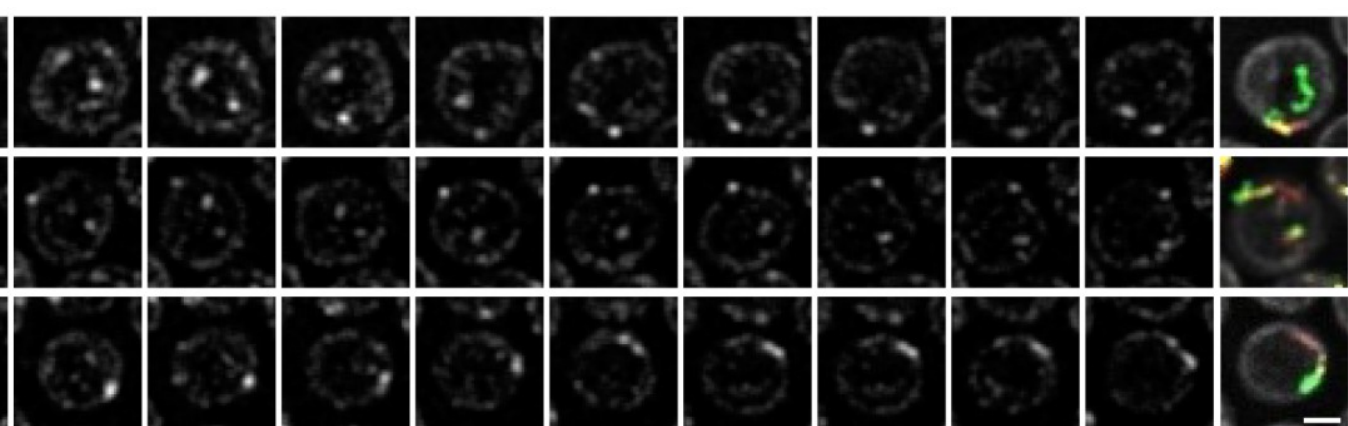

40

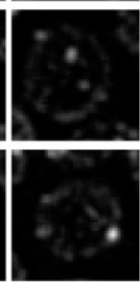

81

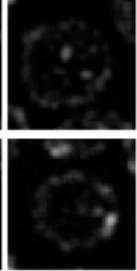

121

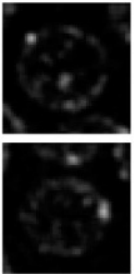

162

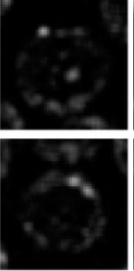

202

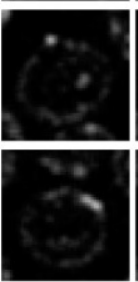

242

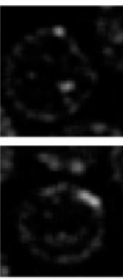

284

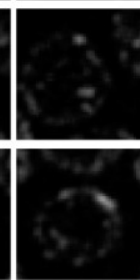

324

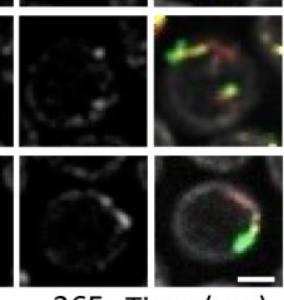

365 Time $(\mathrm{sec})$

Figure 2. Nuclei wriggle in the follicle cells of the Drosophila egg primordia. Importin- $\beta$-GFP pattern in three follicle cell nuclei in St 7 egg primordia. Each picture represents six stacked optical sections, collected in $6.4 \mathrm{~s}$, which include the nucleus. The projection panel (last column) shows maximum intensity streak projections in which the colors correspond to sequential movements that start in green, followed by yellow and finish in red. The speckle patterns are stable over several minutes in the same nucleus and are different in all the nuclei. Scale bar: 2 $\mu \mathrm{m}$.

\section{The speckles moving about are indicators of the wriggling of the entire nucleus}

To determine whether the moving speckles represent drifting rafts in the nuclear envelope, shuffling of the nuclear envelope or wriggling of the entire nuclei, we co-labeled the speckles, the chromatin, and the chromocenter and simultaneously monitored their position over time by confocal time-lapse microscopy. Analysis of the images revealed that the changes in the 
positions of the speckles, the chromatin and the chromocenter are correlated pair wise showing that motions of the speckles represent wriggling of the nuclei (Fig. 3).

To determine whether the endoplasmic reticulum (ER) or part of it also wriggles along with the nucleus, the ER was highlighted by PDI-GFP and the chromatin by Histone2Av-RFP in egg primordia (Fig. 3). It appears that while the nucleus-associated regions of the ER wriggle along with the nucleus, the distant ER parts do not change noticeably during the course of nuclear wriggling. Their minor changes can be accounted for by the constant remodeling of the ER (Bola and Allan, 2009). In fact, the ER may well act as a shock absorber in nuclear wriggling.
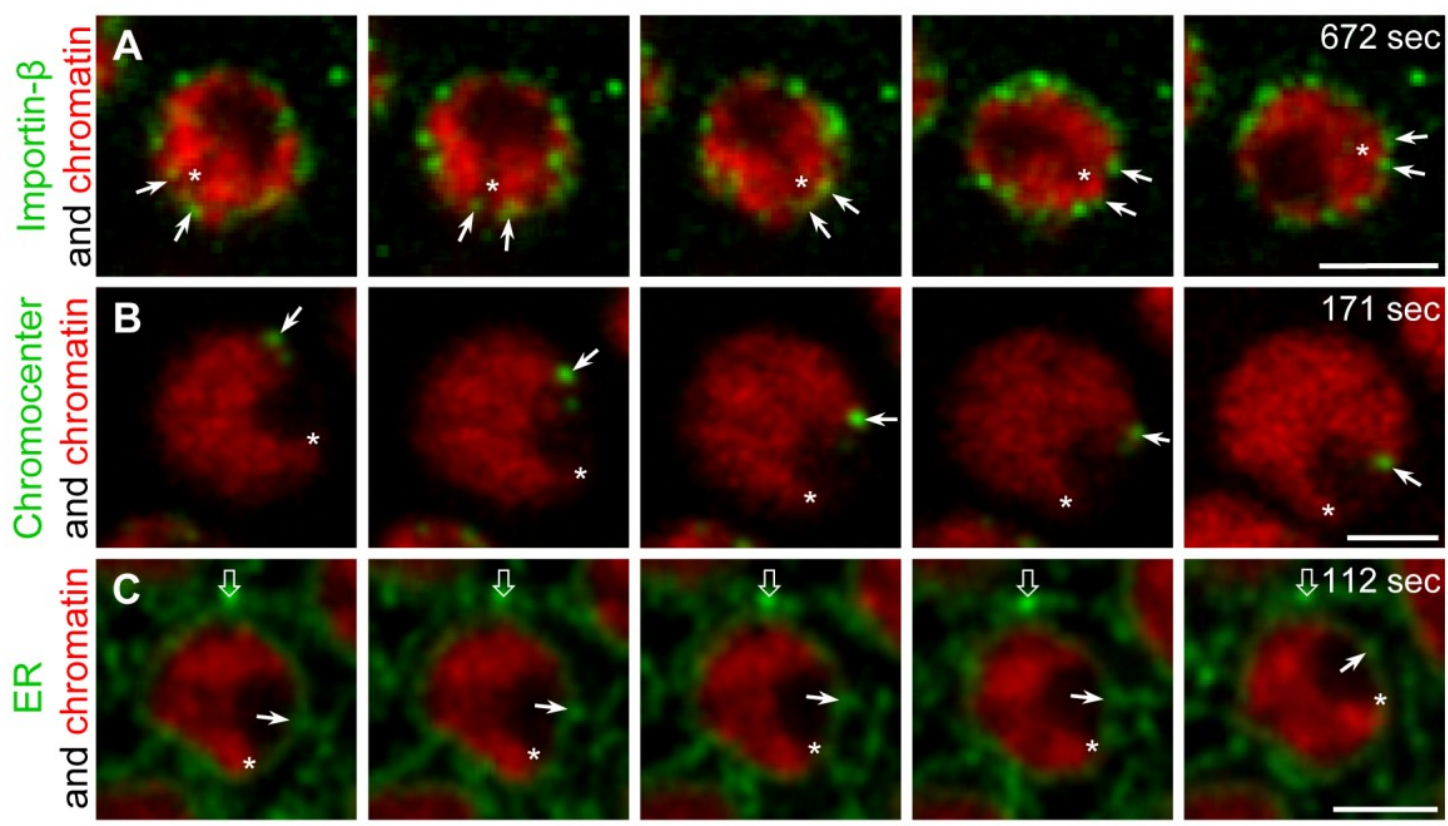

Figure 3. The Importin- $\beta$ speckles, the chromatin, the chromocenter and the endoplasmic reticulum wriggle concurrently. The snapshots illustrate changes in the positions of four cell components in time. The speckles were highlighted by Importin- $\beta$-GFP, the chromatin by Histone2Av-RFP, the chromocenter by CID-GFP and the endoplasmic reticulum by PDI-GFP. The representative optical sections illustrate examples in which the turns occurred in the plane of observation. Arrows point to "landmarks" on the successive optical sections. Chromatin "landmarks" are labeled by *. (A) The chromatin and the nuclear envelope rotated concurrently about $100^{\circ}$ counterclockwise in 672 seconds. (B) The nucleus rotated about $94^{\circ}$ clockwise in 171 seconds. (C) While the part of the endoplasmic reticulum adjoining the nucleus $(\rightarrow)$ rotated about $47^{\circ}$ along with the chromatin, the distant parts ( $)$ stood still. Scale bar: $2 \mu \mathrm{m}$.

\section{Features of nuclear wriggling}

Wriggling of the nuclei denotes sudden twists and slight turns without well-defined axes and extended intermitting resting phases or pauses (Fig. 4). Nuclei in the follicular epithelium wriggle already in the stage 2 egg primordia and cease to do so during stage 9 . The frequency of turning and the angular velocities do not change noticeably during this period. However, the 
peripheral velocities increase along with an increase in the nuclear diameter from about $3.1 \mu \mathrm{m}$ in the stage 2 to roughly $4.7 \mu \mathrm{m}$ in the stage 9 posterior terminal follicle cells (data not shown). In the stage 7 egg chambers, the follicle cell nuclei undergo $2.9 \pm 0.2$ small sudden turns per minute (mean \pm s.d., $\mathrm{n}=61$ ) with an $11.6 \pm 5.8^{\circ}$ angular rotation. While wriggling, the nuclei remain largely spherical and their diameter is about $4 \mu \mathrm{m}$. The average peripheral velocity of wriggling is $1.3 \pm 0.3 \mu \mathrm{m} / \mathrm{min}$ (mean \pm s.d., $\mathrm{n}=22$ ) which corresponds to $34.4 \pm 8.5^{\circ} / \mathrm{min}$ angular velocity) and can exceed $3 \mu \mathrm{m} / \mathrm{min}\left(80^{\circ} / \mathrm{min}\right.$; Fig. 4). These values are in the same range as reported for the wriggling nuclei in the zebra fish embryos and in cultured human nasal mucosa cells (60 and 77\% min; Herbomel, 1999; Pommerat, 1953). The average time of the minor turns is $13.8 \pm 3.7 \mathrm{~s}$ (mean \pm s.d., $\mathrm{n}=35$ ) as was determined from the duration of a number of "one-event" turns (Fig. 4).

The pattern of nuclear wriggling appears to be fully independent in the neighboring cells and shows that the process is cell-autonomous.

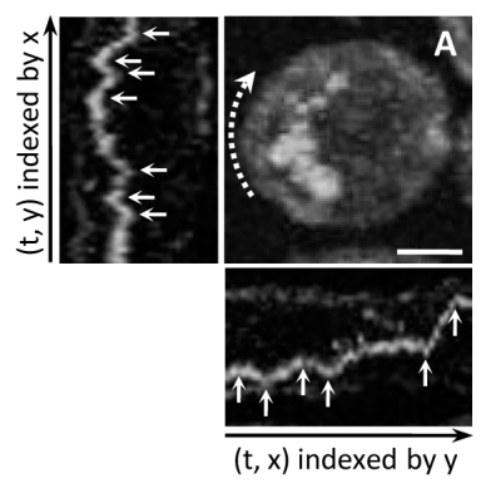

$(t, x)$ indexed by $y$

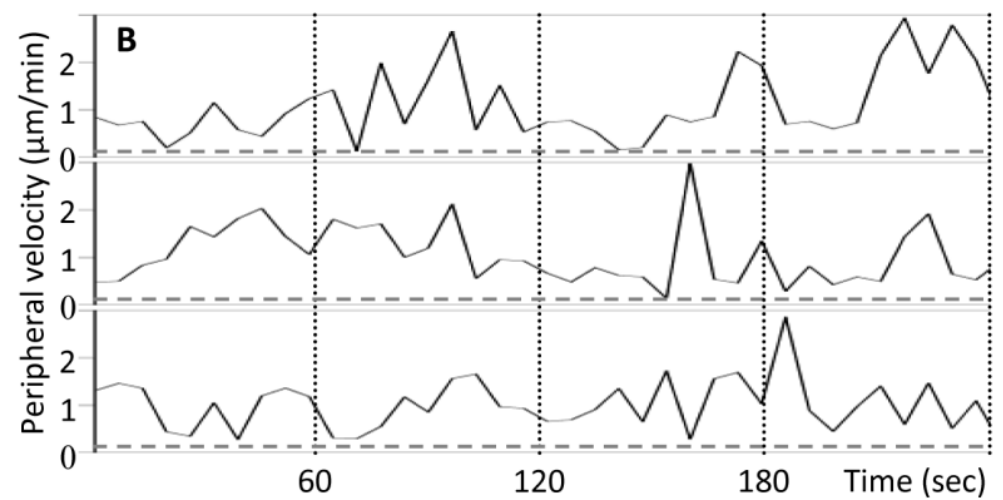

Figure 4. Features of nuclear wriggling. (A) Kymographs illustrating the route of an Importin- $\beta$ GFP speckle in five minutes. Six optical sections that include the nucleus were collected in $6.4 \mathrm{~s}$ and stacked to prepare one picture. The photograph originated by combining 81 such pictures. Arrows indicate some of the sudden changes in the direction and speed of wriggling. (B) Examples illustrating the variations in the peripheral velocity of single speckles in time in three follicle cell nuclei in St 7 egg primordia. The dashed lines define the "base line" at $0.08 \mu \mathrm{m} / \mathrm{min}$ as determined following the elimination of nuclear wriggling by colchicine. The average turning time is $13.8 \pm 3.7 \mathrm{~s}(\mathrm{n}=35)$ as calculated from the duration of "single-event-turns". Scale bar: 2 $\mu \mathrm{m}$.

\section{Nuclei drift along the apical-basal axis}

Until stage 6 of oogenesis, the follicle cell nuclei reside adjacent to the apical membrane with their center at $43 \pm 4 \%$ (mean \pm s.d., $n=32$ ) along the apical-basal axis (Figs. 1 and 5). From stage 6 on, when the formerly cuboidal cells begin to grow and become columnar, the nuclei drift toward the basal membrane at a velocity of about $0.2 \mu \mathrm{m} /$ hour. While they cover an about 5 
$\mu \mathrm{m}$ route in roughly one day, the nuclei reach their final position next to the basal membrane during stage 9 with their centre at $64 \pm 4 \%$ ( $n=34$; Figs. 5 and 7). The difference between the two positions is significant $(\mathrm{P}<0.001)$ and clearly shows that the follicle cell nuclei drift along the apical-basal axis during stages 6 to 9 of egg primordia development.
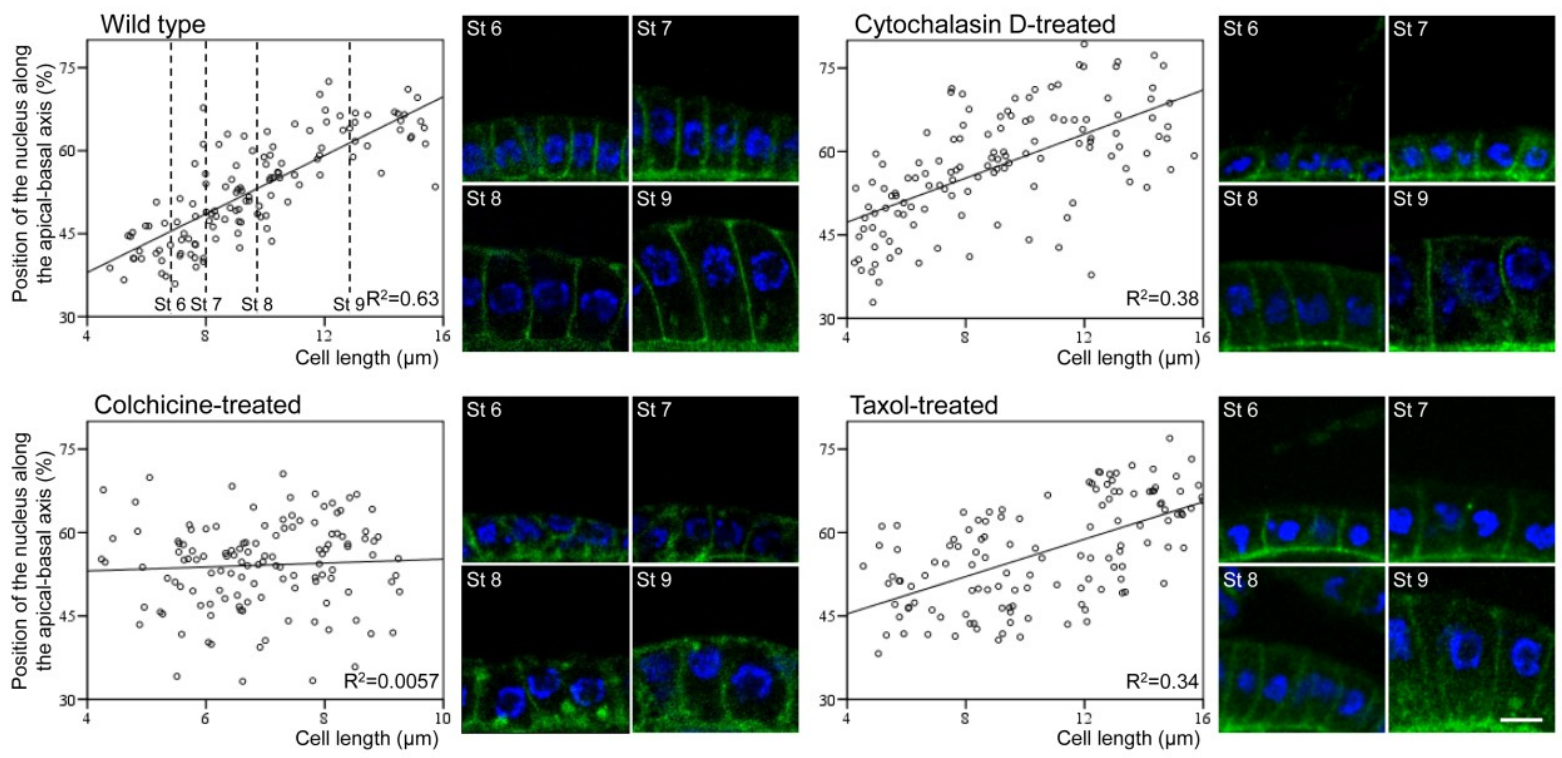

Figure 5. The effect of cytocholasin D, colchicine and taxol on the positioning of the nuclei along the apical-basal axis in the posterior terminal follicle cells of St 6 - St 9 Drosophila egg primordia. Each point in the plots corresponds to one nucleus, and every analysis is based on twelve females. The dashed lines in the wild-type panel show the average lengths of the follicle cells included in the analysis of the St 6 - St 9 egg primordia. When treated, one day old females were injected with $0.3 \mu 125 \mu \mathrm{M}$ cytochalasin $\mathrm{D}, 125 \mu \mathrm{M}$ colchicine or with $1 \mu \mathrm{M}$ taxol solution, and the egg primordia were analyzed eight hours after injection. The pictures were collected from live posterior terminal follicle cells in St 6 - St 9 egg primordia. The cell membrane was highlighted by Spider-GFP and the DNA by Hoechst 33342. Scale bar: $5 \mu \mathrm{m}$.

While the nuclei are drifting, the posterior terminal follicle cells grow immensely, and their volume changes from about 60 to $480 \mu \mathrm{m}^{3}$ (Table 2, Figs. 1 and 5). The commencement of nuclear drifting coincides with the time when mitoses cease and the follicle cells start to become polytenic (Dej and Spradling, 1999; Park et al., 2007). Remarkably, nuclear migration and wriggling come to an end at the same time. The tight correlation between the time of the cessation of wriggling and the fixing of the nuclei at a well-defined position suggests that nuclear wriggling reveals a thus far not described nuclear positioning mechanism. 


\section{In the absence of the actin cytoskeleton, nuclear wriggling speeds up and the nuclei become mispositioned}

The actin cytoskeleton has been reported to be involved in nuclear movements in a number of cell types (von Dassow and Schubiger, 1994; Royou et al., 2002; Chytilova et al., 2000; Dupin et al. 2011). To clarify whether the actin cytoskeleton is engaged in nuclear wriggling and positioning in the Drosophila follicle cells, we disrupted it by cytochalasin D treatment. Two unexpected consequences emerged. (1) The mean angular velocity of wriggling increased from $34.4^{\circ} / \mathrm{min}$ to $78.3^{\circ} / \mathrm{min}$ (Table 1 ). The difference is significant $(\mathrm{P}<0.001)$ and implies that the actin cytoskeleton counteracts nuclear wriggling most likely through increasing cytoplasm viscosity (Goldman, 2002). (2) In the absence of the actin microfilaments that anchor the nuclei at a $64 \%$ position along the apical-basal axis (Yu et al., 2006), the nuclei keep on wriggling and several of them reach the basal membrane, which normally never happens (Figs. 5 and 6).

Table 1. Effects of cytoskeletal drugs and the overexpression of human dynamitin on nuclear wriggling

\begin{tabular}{|c|c|c|c|}
\hline Treatment & $\begin{array}{l}\text { Cell component disrupted, } \\
\text { blocked }\end{array}$ & $\begin{array}{c}\text { Angular velocity } \\
(\% / \mathrm{min})\end{array}$ & Nuclei $^{1}$ \\
\hline Control & None & $34.4 \pm 8.5$ & 22 \\
\hline Cytochalasin $\mathrm{D}(25 \mu \mathrm{M}, 30 \mathrm{~min})$ & Actin cytoskeleton & $78.3 \pm 17.2^{*}$ & 20 \\
\hline Vanadate (15 mM, $30 \mathrm{~min})$ & \multirow{3}{*}{ Dynein } & $39.6 \pm 23.2$ & 31 \\
\hline EHNA (2.5 mM, $30 \mathrm{~min})$ & & $57.2 \pm 47.4$ & 19 \\
\hline Overexpressed human dynamitin & & $35.9 \pm 17.0$ & 48 \\
\hline Lidocaine $^{2}$ (50 mM; $\left.10 \mathrm{~min}\right)$ & Kinesin, MTs & $2.9 \pm 1.1^{2}$ & 12 \\
\hline Colchicine $(125 \mu \mathrm{M}, 30 \mathrm{~min})$ & \multirow{2}{*}{ MTs } & $2.1 \pm 0.7 *$ & 20 \\
\hline Taxol (1 $\mu \mathrm{M}, 30 \mathrm{~min})$ & & $1.9 \pm 1.0^{*}$ & 20 \\
\hline Cytochalasin D + colchicine & MTs, actin cytoskeleton & $1.9 \pm 0.9 *$ & 20 \\
\hline
\end{tabular}

${ }^{1}$ Number of analyzed posterior terminal follicle cell nuclei representing at least six stage 7 egg primordia in 3 females.

${ }^{2}$ For nuclei affected by lidocaine.

* Significantly different from the control at $\mathrm{P}<0.001$. 
Table 2. Volume of the posterior terminal follicle cells

\begin{tabular}{|l|c|c|}
\hline \multirow{2}{*}{ Treatment $^{1}$} & \multicolumn{2}{|c|}{ Cell volume $\left(\mu \mathrm{m}^{3}\right)$} \\
\cline { 2 - 3 } & Stage 6 & Stage 9 \\
\hline Control & $62 \pm 16$ & $480 \pm 129$ \\
\hline Cytochalasin D $(25 \mu \mathrm{M}, 30 \mathrm{~min})$ & $95 \pm 20$ & $461 \pm 128$ \\
\hline Colchicine $(125 \mu \mathrm{M}, 30 \mathrm{~min})$ & $75 \pm 16$ & $220 \pm 28^{*}$ \\
\hline Taxol $(1 \mu \mathrm{M}, 30 \mathrm{~min})$ & $66 \pm 16$ & $477 \pm 168$ \\
\hline
\end{tabular}

${ }^{1}$ The drugs were injected into female abdomens and the egg primordia analyzed eight hours following the injections. Six cells were analyzed for each set of data. Stages of the egg primordia were determined based on the size ratio of the oocyte and its daughter cells (cf. King 1970).

* Significantly different from the control at $\mathrm{P}<0.05$.

While cytochalasin D does not influence the growth of the follicle cells, the positioning of the nuclei differs in the wild-type and the treated cells (Fig. 5; $\mathrm{P}=0.024$; GraphPad Prism). The difference comes mainly from the drifting of several of the nuclei to the basal membrane (Fig. 5 and 6). Evidently, the forces that make the nuclei wriggle and drift continue their action in the absence of the microfilaments and bring the nuclei to unusual positions near the basal membrane. The present observation is in line with the previously described function of the actin microfilaments, i.e., fixing the nuclei at their final position (Yu et al., 2006).
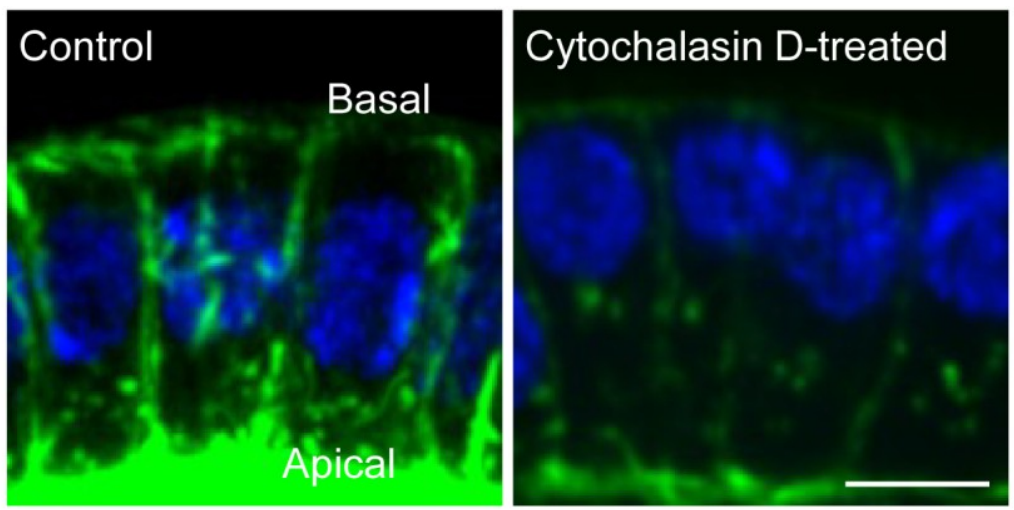

Figure 6. Following the elimination of the actin microfilaments by a $30 \mathrm{~min}$ cytochalasin D treatment, several of the follicle cell nuclei drift near the basal membrane. Actin was highlighted by actin-GFP and the DNA by Hoechst 33342. The optical sections represent St 9 follicle cells. Scale bar: $5 \mu \mathrm{m}$. 


\section{MT motor functions do not seem to be required for nuclear wriggling}

Cytoplasmic dynein has been shown to be engaged in nuclear positioning and acts largely through three mechanisms. (1) The MTOC, tightly associated with the nucleus through dyneins, nucleates the astral MTs which, while growing and pushing against the cell membrane, generate the force required to position the nucleus (reviewed in Reinsch and Gönczy, 1998; Gönczy et al., 1999; Morris, 2003; Tolić-Nørrelykke, 2008 and 2010; Zhao et al. 2012). The process is reminiscent of the growing interpolar MTs that push apart the daughter centrosomes (Venkei et al., 2006). (2) In a number of higher eukaryotic organisms, dynein molecules anchored to the cell cortex pull on the astral MTs and move the nucleus to its proper position (reviewed in Dujardin and Vallee, 2002; Allan, 1996). (3) In some instances, the dynein motors are linked to the nuclear envelope and, while moving along the MTs, transport the nucleus to its destination (Rouviere et al., 1994; Schatten, 1994; Allan, 1996).

To determine whether dyneins are involved in nuclear wriggling in the follicle cells, we inhibited dynein functions by vanadate or EHNA treatments and also by overexpressing dynamitin in the follicle cells to block dynein-dependent movements (Reinsch and Karsenti, 1997; Bouchard et al., 1981). Neither vanadate nor EHNA treatment resulted in significant changes in the speed of nuclear wriggling $(\mathrm{P}>0.05$; Table 1$)$ indicating that the process is dynein independent (Table 1). In accordance with the results of the vanadate and EHNA treatments, the overexpression of dynamitin did not perceptibly alter nuclear wriggling (Table 1). The lack of effect of the above treatments on nuclear wriggling is unlikely to be the consequence of improper treatment since abnormal mitoses were apparent in the follicle cells of the egg primordia younger than stage 6. Instead, it shows that cytoplasmic dynein does not play a perceptible role in nuclear wriggling.

Kinesins have also been shown to be involved in certain types of nuclear positioning. For example, the Kar3p kinesin motor is required during mating in $S$. cerevisiae to bring the two nuclei together prior to their fusion (reviewed in Rose, 1996). To clarify the role of kinesins in nuclear wriggling, we made use of lidocaine, which has been known to reversibly inhibit MTbased kinesin motility (Miyamoto et al., 2000). A $50 \mathrm{mM}$ lidocaine treatment for 10 minutes blocked nuclear wriggling in about $70 \%$ of the follicle cells, and the angular velocity dropped significantly (from $34.4 \pm 8.5$ to $2.1 \pm 1.1 \%$ min; $P<0.001$, Table 1 ). Analysis of the MTs highlighted by EB1-GFP on their growing end revealed that the MTs did not grow in the affected follicle cells; instead, the EB1-GFP label spread all along the MTs indicating disturbed MT organization and/or function. The remaining $30 \%$ of the lidocaine-treated follicle cells seemed largely unaffected: the MTs were growing with the EBI-GFP labeling towards their plus ends 
and the nuclei were wriggling. The blocking effect vanished without washing the lidocaine away, and the wriggling of the stalled nuclei resumed after about 20 minutes. Although the above results may indicate that kinesins are involved in nuclear wriggling, its effects on nuclear wriggling may well stem from disturbed MT function since lidocaine has been known to alter MT dynamics (Lother et al., 1979). The assumption that kinesins are unlikely to be involved in nuclear wriggling is further supported by the finding that RNAi-silencing of the functions of 14 of the 25 Drosophila kinesin-encoding genes in the follicle cells had no perceptible outcome on nuclear wriggling (Table 3).

\section{Dynamic MTs render the nuclei wriggling}

The above results raised the possibility that MTs are responsible for nuclear wriggling. To test this possibility, we treated egg primordia with colchicine to disrupt the MTs. There was no sign of nuclear wriggling in any of the studied follicle cells, demonstrating that nuclear wriggling is MT dependent (Table 1). In fact, nuclear wriggling was absent following a combined treatment of the egg primordia with both cytochalasin D and colchicine (Table 1). The slight trembling of the nuclei following colchicine treatment represents the "base line" of the system and was used to determine the average time of turning of the nuclei (Fig. 4).

Table 3. Effect of kinesin disruption by RNAi constructs

\begin{tabular}{|l|c|c|c|c|c|}
\hline Kinesin & Transformant ID & CG number & Off targets ${ }^{2}$ & $\begin{array}{c}\text { Mean angular velocity } \\
\text { (\%/min) } \\
\text { (Mean } \pm \text { s.d.) }\end{array}$ & Nuclei $^{1}$ \\
\hline CENP-ana & 49776 & CG33694 & 2 & $29.8 \pm 8.7$ & 20 \\
\hline CENP-meta & 35081 & CG6392 & 0 & $32.2 \pm 4$ & 20 \\
\hline CG9913 & 36461 & CG9913 & 0 & $29.8 \pm 5.2$ & 25 \\
\hline KHC & 44338 & CG7765 & 2 & $26.5 \pm 10$ & 20 \\
\hline Kinesin-73 & 24226 & CG8086 & 1 & $34.5 \pm 5.3$ & 20 \\
\hline Klp3A & 35974 & CG8590 & 0 & $34.9 \pm 8.2$ & 10 \\
\hline Klp38B & 31330 & CG10718 & 0 & $28.7 \pm 8.4$ & 12 \\
\hline Klp59C & 48576 & CG3219 & 0 & $33.1 \pm 5.8$ & 13 \\
\hline Nod & 48148 & CG1763 & 0 & $28.6 \pm 6$ & 5 \\
\hline Pavarotti & 46134 & CG1258 & 0 & $28.1 \pm 6.7$ & 20 \\
\hline Klp61F & 52549 & CG9191 & 1 & $31.3 \pm 6.6$ & 20 \\
\hline Klp68D & 27943 & CG7293 & 0 & $33.8 \pm 7.1$ & 20 \\
\hline Klp53D & 47172 & CG8566 & 1 & $31.9 \pm 8.6$ & 20 \\
\hline Klp10A & 41534 & CG1453 & 0 & $35.2 \pm 7.3$ & 20 \\
\hline
\end{tabular}

${ }^{1}$ The number of follicle cell nuclei that represent at least 6 egg primordia in 3 females.

${ }^{2}$ Off-target effects arise when an introduced RNA - to induce RNA1 - has a base sequence that can pair with and thus reduce the expression of multiple genes at a time. 
Positioning of the nuclei may well depend on the presence of intact MTs (and their action to poke and make the nuclei wriggle) as indicated by the finding that the nuclei do not wriggle and are not positioned in the colchicine treated follicles: the position of the nuclei is random following an eight-hour colchicine treatment (Fig. 5; P $<0.0001$, GraphPad Prism). However, the issue of wriggling and nuclear positioning is complicated by the fact that the follicle cells remain small in the long-term colchicine-treated cells (Table 2, Fig. 5).

To further elaborate on the relationship between the wriggling and the drifting of the nuclei, we transferred egg primordia into a $125 \mu \mathrm{M}$ colchicine solution for $30 \mathrm{~min}$ and analyzed the follicle cells afterwards. The MTs were highlighted by Jupiter-GFP and the nuclei by Hoechst 33342. Under such conditions, practically $100 \%$ of the nuclei are positioned adjacent to the basal membrane in the stage 7 follicle cells (Fig. 7). The phenomenon is most likely related to a faster diffusion of the colchicine into the basal cell region and the destruction of the MTs there as compared to the apical cell regions where some of the MTs were still present (Fig. 7). The apically nucleated MTs make the nuclei wriggle and achieve their rapid drifting to the basal membrane. The same treatment of stage 9 egg primordia had no effect on the position of the nuclei that had already been fixed at the characteristic $64 \%$ position along the apical-basal axis (Fig. 7). Obviously, the microfilaments do not yet fix the nuclei in the stage 7 follicle cells.

Under the above conditions and highlighting the MTs using tubulin-GFP, the $125 \mu \mathrm{M}, 30 \mathrm{~min}$ colchicine treatment eliminated all the MTs; the nuclei stopped wriggling and failed to drift along the apical-basal axis (Fig. 7). The different colchicine sensitivity of the MTs as highlighted by Jupiter-GFP and tubulin-GFP may well be related to the protective activity of the Jupiter-GFP and/or the sensitizing effect of the tubulin-GFP molecules on the MTs.

Nuclear wriggling also stopped following the treatment of the ovarioles with low doses of taxol, a drug that inhibits MT dynamics without causing their disassembly (Table 1). Although the volume of the taxol-treated follicle cells did not differ from that of the control (Table 2), the effect of taxol on nuclear positioning was apparent (Table 1, Fig. 5; $\mathrm{P}=0.0009$, GraphPad Prism). A 30 min treatment with $1 \mu \mathrm{M}$ taxol eliminated both the wriggling and the drifting of the nuclei. The above described correlation between the presence of dynamic MTs and nuclear wriggling and drifting supports a causal correlation between the two processes. 


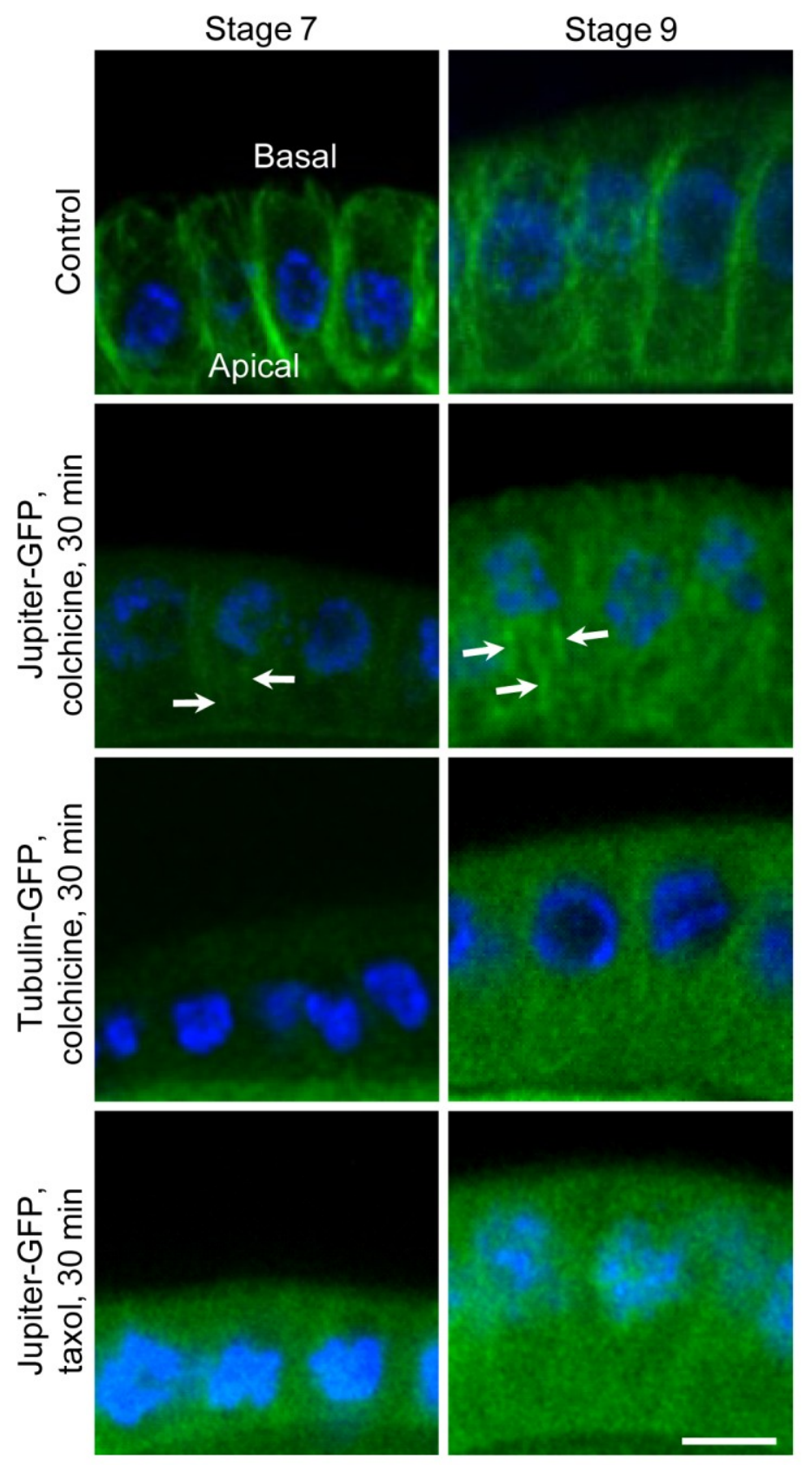

Figure 7. Position of the follicle cell nuclei along the apical-basal axis. Nuclei reach the vicinity of the basal membrane following a $30 \mathrm{~min}$ colchicine treatment of St 7 egg primordia. Arrows point at some of the MTs that persist in the apical cell region. (Upon destruction of the MTs, some of the Jupiter-GFP enters the nucleus; cf. Karpova et al., 2006) The tubulin-GFPhighlighted MTs decompose in the presence of colchicine, the nuclei do not wriggle and fail to drift. Nuclei stop wriggling and drifting following taxol treatment. The pictures represent stacked optical sections that include the nucleus. The nuclei were labeled by Hoechst 33342. Scale bar: 5 $\mu \mathrm{m}$.

\section{MTs in the follicle cells}

To visualize the MTs in the follicle cells, we highlighted them using Jupiter-GFP (Fig. 8).

Apparently, there is no sign of a well defined MTOC in the follicle cells from stage 6 on when 
they start to become polytenic (Park et al., 2007) and start to drift along the apical-basal axis. However, as anti- $\gamma$-tubulin labeling revealed, there is a profound cortical enrichment of the MTOCs that may well seed MTs throughout the egg cortex (Fig. 8). In general, the organization of the MTs in Drosophila follicle cells is very similar to that found in other polarized epithelial cell types, in which the MTs appear as prominent linear, non-centrosomal arrays along the apical-basal axis with the minus ends apically and the plus ends basally (Fig. 8; cf. Bacallao et al., 1989; Clark et al., 1997). Highlighting the growing ends of the MTs using EB1-GFP clearly showed that MTs do indeed grow from non-MTOC seeds embedded in the cortex of the follicle cells and - reported here for the first time - some of the MTs grow from the basal membrane region toward the nucleus (Fig. 8). A detailed analysis of time-lapse optical sections of three posterior follicle cells in stage 7 egg primordia revealed 36, 29 and 39 EB1-GFP-highlighted MTs growing from the apical toward the basal region and 14, 21 and 11 MTs growing from the opposite direction; thus, about 2.3-times more MTs are nucleated in the apical than in the basal cell cortex.

\section{Dynamic MTs exert force on the nuclei}

To analyze the interaction between the MTs and the nuclei, we constructed egg primordia in which the NPCs were highlighted by Importin- $\beta$-GFP and the MTs by Jupiter-GFP. Follicle cells of early stage 9 egg primordia were examined, in which the nucleus/cell volume ratio is rather low providing appropriate conditions to analyze the interactions. Long series of optical sections were collected in time, such that five sections - recoded in $4.6 \mathrm{~s}$ - spanned a nucleus. Apparently, only the tips of the MTs interact with the nucleus, and the interactions are transient; the average dwell-time is $15.9 \pm 7.5 \mathrm{~s}$ (mean \pm s.d., $\mathrm{n}=35$; Fig. 9), slightly longer than the $13.8 \mathrm{~s}$ during which the nuclei accomplish their minor turns. It appears that the MTs interact with the nucleus and exert forces through the NPCs (Figs. 9 and 10). This may well be the case since some of the nucleoporins have been known to interact with both Importin- $\beta$ and the MTs (Tirian et al., 2003). The analysis of the optical sections further revealed that several of the growing MTs may poke the same nucleus at the same time (Fig. 9). 

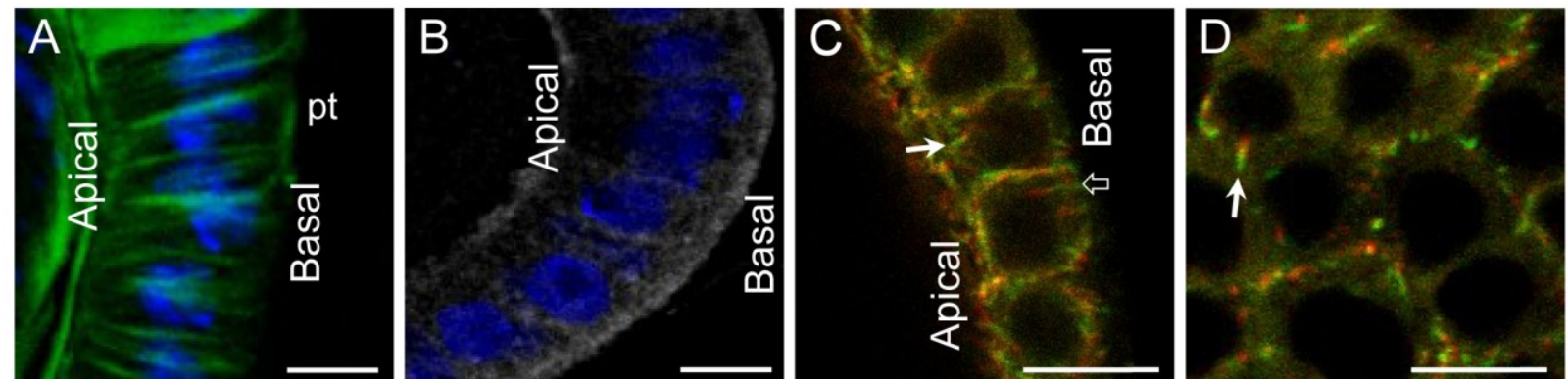

Figure 8. MTs in the posterior terminal follicle cells. (A) Horizontal, stacked optical sections that include the follicle cells in a stage 8 egg primordium. The prominent linear, noncentrosomal MT-arrays - as highlighted by Jupiter-GFP - align along the apical-basal axis. Note the lack of well-defined MTOCs. (B) Anti- $\gamma$-tubulin antibody staining (in grey) revealed cortical enrichment of $\gamma$-tubulin throughout the cell cortex. The nuclei appear in blue. (C and D) Time-lapse projections of EB1-GFP-highlighted MT ends in follicle cells of St 7 egg primordia. The colors represent sequential movements that start in green followed by yellow and finish in red. In (C), ten longitudinal optical sections - recorded in $23 \mathrm{~s}$ - were merged. Although 2.3-times more MTs originate in the vicinity of the apical membrane and grow along the apical-basal axis $(\rightarrow)$, there are MTs nucleating in the basal membrane region and growing toward the apical membrane $(\diamond)$. In (D), six cross optical sections - recorded in $13.8 \mathrm{~s}$ - were merged. The arrow points to an MT that was seeded in the lateral cell cortex and grew toward the nucleus. The MTs grew about 1.2 $\mu \mathrm{m}$ per minute, a speed that fits well with the reported in vivo growth rate of the MTs (cf. Laan and Dogterom 2010). The pt symbol stands for posterior terminal follicle cells. Scale bar: $5 \mu \mathrm{m}$.

Following bumping into the nuclei, the MTs tend to buckle showing that the nuclei resist while the MTs continue to grow (Figs. 9 and 10). The MTs gradually bend into an arch as a consequence of their polymerization against the nuclear envelope. During the minor turns, the MTs maintain continuous interaction with both the cell cortex and the nuclear envelope (Figs. 9 and 10). MTs poking the nuclei may well exert forces as described earlier for growing MTs that contact cellular structures (Inoue and Salmon, 1995; Li, 2008; Laan and Dogterom 2010; Zhao et al. 2012). Thus, it appears that nuclear wriggling is the result of the activity of dynamic MTs that keep on poking the nuclei and make them wriggle.

It is important to note that although the migration of the follicle cell nuclei ceases during stage 9 of egg primordia development (when microfilaments anchor the nuclei at the $64 \%$ position along the apical-basal axis), poking of the nuclei by the growing MTs continues during the rest of egg primordia development, and its features do not change perceptibly. 


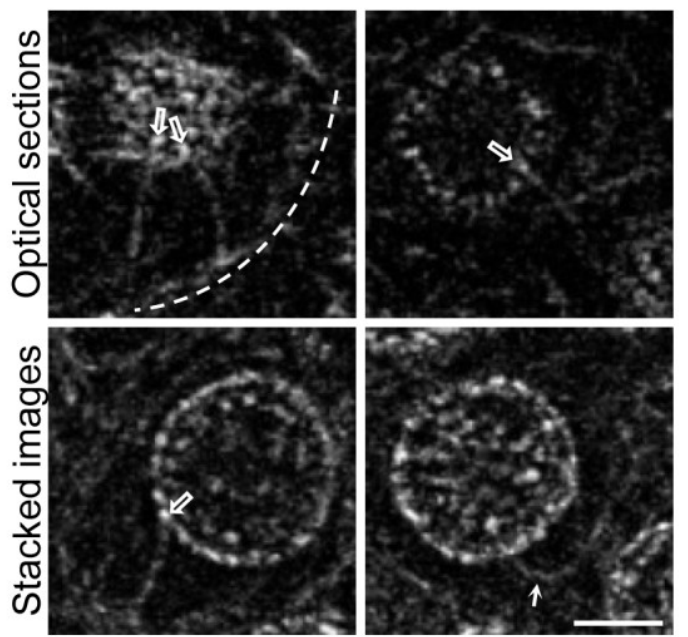

Figure 9. The MTs and the nuclei interact in the follicle cells. Importin- $\beta$-GFP highlights the NPCs and Jupiter-GFP the MTs in follicle cells of stage 9 egg primordia. The $\mathrm{Z}$ projections show that several MTs may contact the same nucleus at the same time. Some of the MTs buckle $(\uparrow)$ showing that they exert force on the nucleus. The optical sections reveal that the MTs originate from the cell cortex (outlined by a dashed line) and not from a well-defined MTOC. It appears that the MTs establish contact with the NPCs (§). Scale bar: $2 \mu \mathrm{m}$.
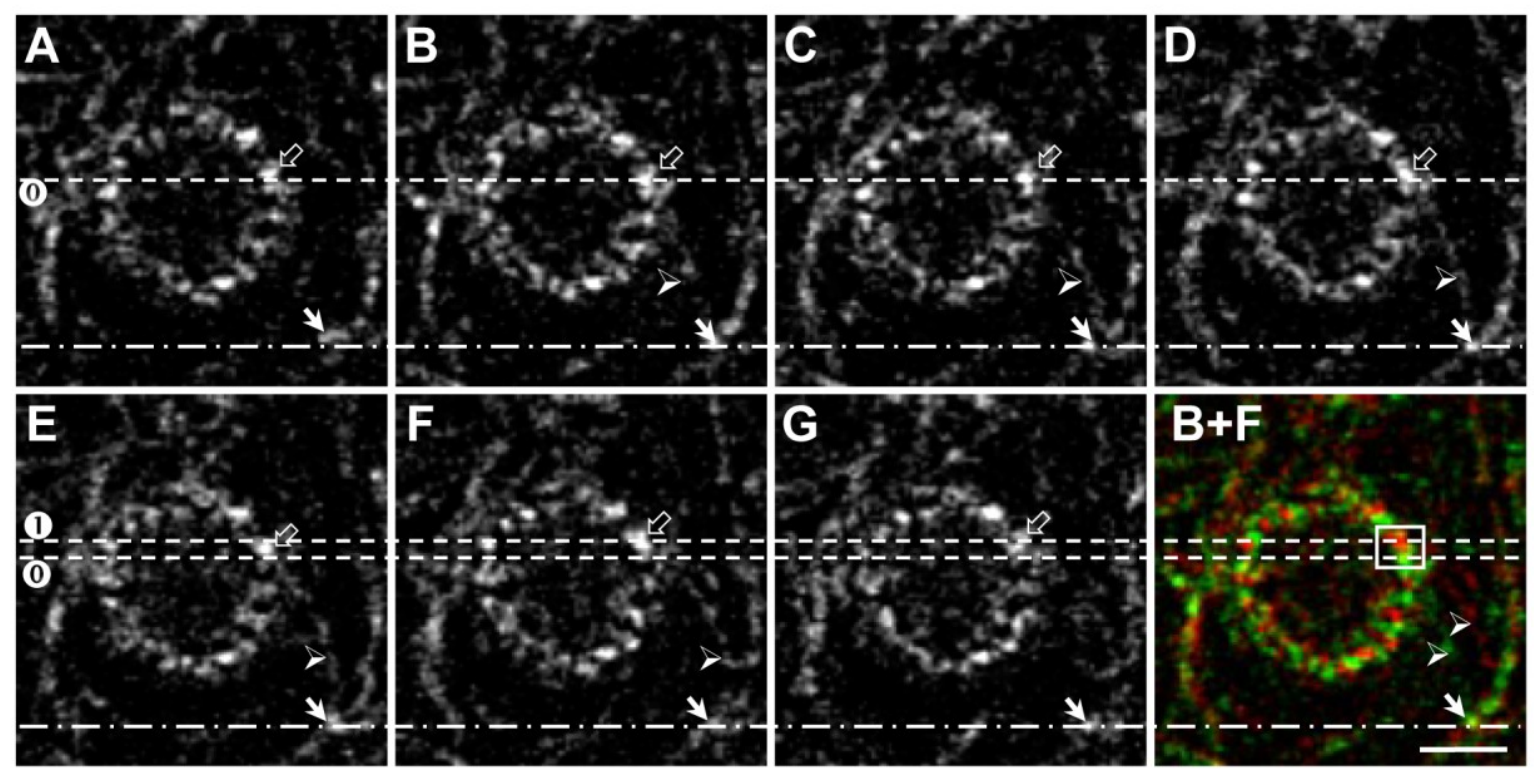

Figure 10. Nuclear wriggling is the outcome of the polymerization force exerted by the MTs. Seven successive optical sections (A-G) were recorded in $17 \mathrm{~s}$ from a follicle cell in a St 9 egg primordium. Importin- $\beta$-GFP specs delineate the nuclear envelope and Jupiter-GFP the MTs. Arrows $(\searrow$ ) point to an MT seed in the cell cortex. (Note that its position did not change throughout the recording, as indicated by the dash-dot-dash line.) An MT bumps into an NPC in the nuclear envelope $(\backsim)$. The MT bends $(\triangleright)$ indicating a pushing force exerted on the nucleus. Buckling of the MTs, as a rule, is followed by their disassembly $(\mathrm{G})$. The pushing force brings about a slight turn of the nucleus as indicated by the two dashed lines ( $\mathbf{O}$ and $\mathbf{0})$ that illustrate the change in the position of the NPC. In (B+F), the second (B in green) and the sixth (F in red) optical sections were merged to illustrate the change in the position of an NPC (framed box). Scale bar: $2 \mu \mathrm{m}$.

\section{Growing MTs tuck in the nuclear envelope}

While analyzing follicle cell nuclei in which the NPCs were highlighted by Importin- $\beta$-GFP and the chromatin by Histone2Av-RFP, we noticed that every so often a group of NPCs appear 
inside the otherwise ball-shaped nuclei in the younger than stage 6 egg primordia, as if the nuclear envelope was tucked into the inside of the nucleus (Fig. 11). Remarkably, such NPCs never appear inside the follicle cell nuclei beyond the $6^{\text {th }}$ stage of egg primordia development. Moreover, the phenomenon never appears in the younger than 6 egg primordia kept in colchicine solution. These observations reveal an MT-associated phenomenon and perhaps the "hardening" of the nuclear envelope in the follicle cells of the stage 6 egg primordia. (Similar MT-associated fluctuations were reported for nuclei in the early Drosophila embryos recently; Hampoelz et al. 2011.)
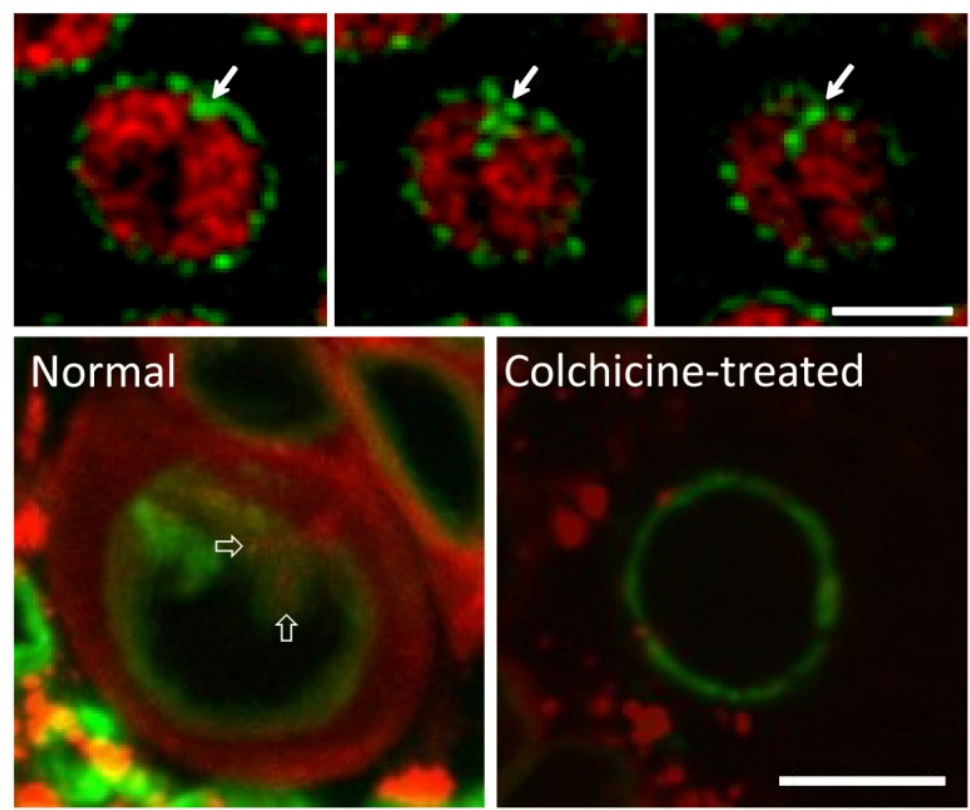

Figure 11. Poking MTs can indent the nuclear envelope. The three optical sections on the upper panel show the nucleus of a follicle cell in a stage 5 egg primordium in which some of the Importin- $\beta$-GFP-highlighted NPCs appear among the Histone2Av-RFP-labeled chromatin (arrows). (The three optical sections were collected in $8.8 \mathrm{~s}$ in the same plane of recording. Each of the lower panel pictures show 50 stacked optical sections recorded in $134 \mathrm{~s}$. They were collected on neuroblasts dissected from a late third instar larva in which an Act5C-Gal4 driver ensured the production of both EB1-Cherry, to label the growing end of the MTs, and laminGFP, to highlight the nuclear lining. The creases of the nuclear envelope are filled with MTs (仓). The creases are absent following colchicine treatment, and the nuclei appear ball-shaped. Scale bar: $5 \mu \mathrm{m}$.

To decide whether the MTs tuck in the nuclear envelope with the NPCs or perhaps even sever it, we simultaneously highlighted the lining of the nuclear envelope by lamin-GFP and the growing ends of the MTs by EB1-mCherry. Driving the UAS-Lamin:GFP and the UASEB1:mCherry transgenes by either of the follicle cell-specific drivers slbo-Gal4 or e22c-Gal leads to the formation of a red, amorphous EB1-Cherry halo around the nuclei and makes the 
analysis impossible. The ubiquitous Act5C-GAL4 and Tub-GAL4 or the T155-Gal4 (follicle cellspecific) drivers bring about death during late larval and/or early pupal stages. However, the central nervous system (CNS) can be dissected, e.g., from the Act5C-GAL4; UAS-Lamin:GFP; $U A S-E B 1: m C h e r r y$ larvae, and the neuroblasts inside are amenable to analysis. Apparently, the nuclei of the neuroblasts do not wriggle; instead large creases - filled with EB1-highlighted MTs - penetrate from the nuclear envelope into the inside of the nuclei (Fig. 11). Creases never form when the larval CNS is incubated in colchicine solution indicating that forces exerted by the growing MTs induce the formation of the creases (Fig. 11).

\section{DISCUSSION}

Positioning of the nucleus has been known to be a carefully regulated process, e.g., in asymmetric cell divisions, formation of the neuromuscular junctions and cell migration (see Tolic-Nørrelykke, 2010; Dupin and Etienne-Manneville, 2011 for recent reviews). In this twostep process, the nucleus is first moved to its proper position and then fixed with anchoring filaments. Both steps depend on the cytoskeleton (Dupin et al. 2011). Forces that needed to position the nuclei are generated either by the molecular motors, kinesins and dyneins, or by the polymerizing components of the cytoskeleton. The so-called polymer motors rely on the polymerization and depolymerization of dynamic biopolymers (reviewed in Mogilner and Oster, 2003; Laan and Dogterom, 2010; Inoue and Salmon, 1995). Force generation by the self assembling linear filaments is based on a so-called polymerization ratchet: the addition of new tubulin dimers into the growing filament provides energy for mechanical work. Polimerization takes place in contact with the barrier on which a load is applied. MTs, the most rigid cytoskeletal components, possess dynamic instability, an intrinsic ability to rapidly switch between growing and shrinking. While growing or shrinking, the MTs have been known to exert forces and can thus move cell organelles (Brouhard and Hunt, 2005; Venkei et al., 2006; reviewed in Tolić-Nørrelykke 2010; Dupin and Etienne-Manneville, 2011). Dynamic instability allows the MTs to search the intracellular space, to dis- and reassemble into different arrangements and temporarily interact with cellular components (Howard 2006). MTs grow practically straight and behave as homogeneous elastic rods (Dogterom 1997). When the MTs reach a barrier in the cell, their growth rate decreases to about $0.2 \mu \mathrm{m}$ per minute and they frequently buckle before disassembling (Brangwynne et al. 2007; Li 2008; Laan \& Dogterom 2010). Buckling is a clear sign of force generation by the growing MTs and - as described in the present paper - the growing MTs that bump into the nuclei in the follicle cells of the Drosophila 
egg primordia buckle and bring about minor turnings of the nuclei. MTs poking the follicle cell nuclei make them wriggle, i.e., they carry out about three sudden and random minor turns in a minute with about $12^{\circ}$ angular rotations. The fact that the process does not take place in the presence of colchicine or taxol, agents that interfere with the formation or function of the MTs, shows best that wriggling is indeed the outcome of the growing MTs.

\section{Scenario of nuclear wriggling}

MTs are seeded throughout the cell cortex and grow into the interior of the follicle cells (Fig. 8). MTs that grow and bump into the nuclear envelope contact it - as it appears - through the NPCs for about 16 seconds (Figs. 9 and 10). Although the growing MTs exert forces on the nuclear envelope from the moment of contacting it, buckling becomes perceptible only after about one second, and the turning of the nucleus begins after roughly an additional second (Fig. 10). The MTs can not overcome the stiffness of the nuclear envelope, and the force they generate during this short period of time "serves" mostly to overcome the resistance of the endoplasmic reticulum and the viscosity of the cytoplasm. Buckling becomes pronounced in time and disappears when the MTs disassemble. By this time, the nuclei accomplish slight turns that last for about 14 seconds (Figs. 10 and 12).

$F_{c}$, the buckling force exerted on the nuclear envelope by the MTs, was estimated by considering that $F_{c}$ is inversely proportional to the square of the MT length $(L): F_{c}=\pi^{2} E I / L^{2}$, where $E I$ stands for flexural rigidity with values ranging from 4 to $40 \times 10^{-24} \mathrm{Nm}^{2}$ (Elbaum et al., 1996; Felgner et al., 1997; Mickey and Howard, 1995; Dogterom and Yurke, 1997). Considering that the size of the MTs that poke the nuclei in the follicle cells varies between 2.5 and $6.5 \mu \mathrm{m}$ and taking flexural rigidity $25 \times 10^{-24} \mathrm{Nm}^{2}$, the buckling force appears to vary between 40 and 5 piconewtons, respectively. Similar values were reported, e.g., for the MTs that push the oocyte nucleus during its positioning in the developing Drosophila egg primordia (Inoue and Salmon, 1995; Dogterom and Yurke, 1997; Janson et al., 2003; Zhao et al. 2012). 

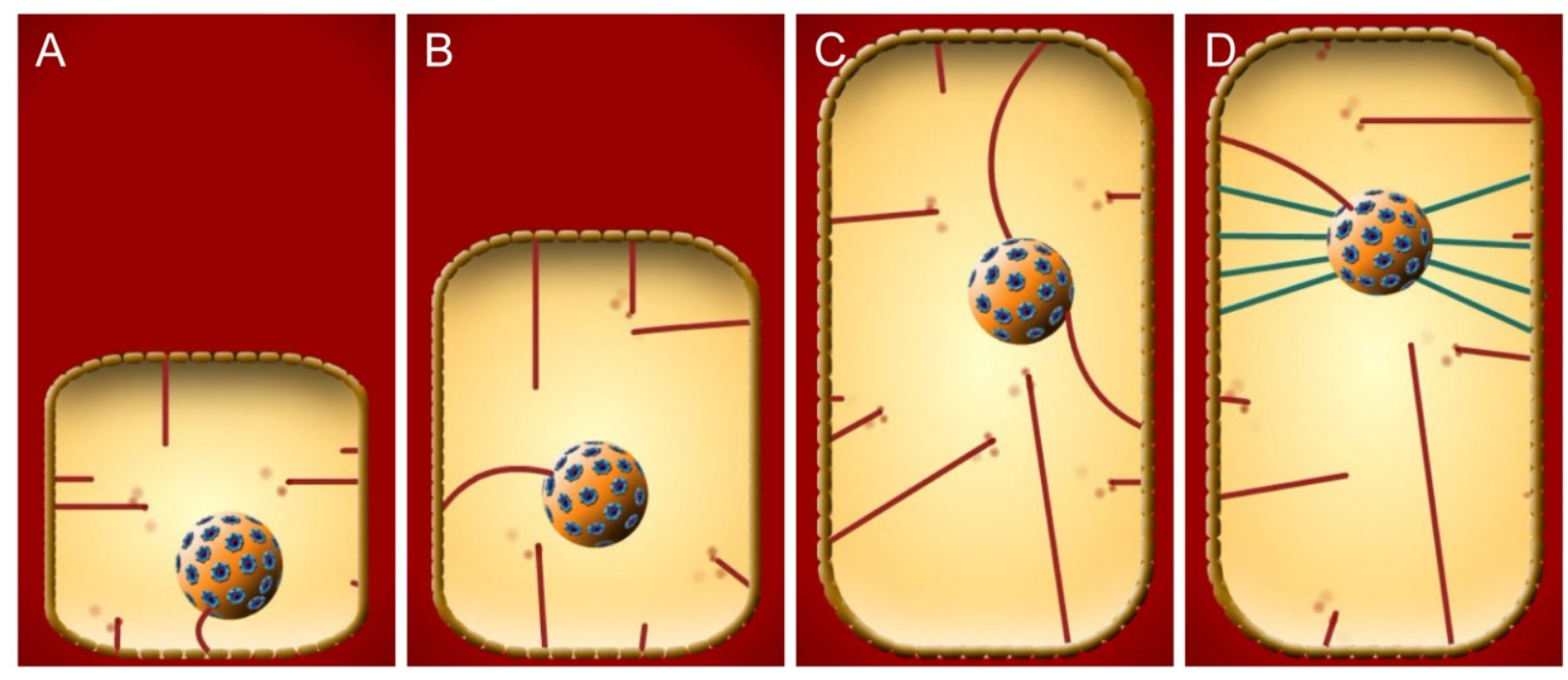

Figure 12. Scenario of nuclear wriggling and positioning in Drosophila follicle cells. (A) Tips of the dynamic MTs bump into the nuclear envelope. Due to polymerization, the MTs buckle and exert pushing forces as the force exceeds their flexural rigidity $(B, C)$ When cells start to become columnar the nuclei - while wriggling - drift away from the apical cell region along the apicalbasal cell axis to the vicinity of the basal membrane. (D) Their positioning is terminated once become anchored by actin filaments (labeled blue) during stage 9.

Although the MTs keep on poking and make the follicle cell nuclei wriggle already in the very young egg primordia, the nuclei do not drift in the yet dividing, cuboidal cells. When, however, the cells begin endoreplication and start to become columnar (Horne-Badovinac and Bilder, 2005) the nuclei - while wriggling - drift away from the apical cell region along the apical-basal cell axis to the vicinity of the basal membrane (Fig. 1). Their voyage is terminated once they arrive at their final position and become anchored by actin filaments during stage 9 (Yu et al., 2006). As an outcome of wriggling, the nuclei become repositioned whilst they cover an about 5 $\mu \mathrm{m}$ route in about a day.

We perceive that those MTs that originate in the lateral egg cortex and poke the nuclei keep them along the apical-basal cell axis during drifting. The most plausible explanation for the drifting of the nuclei along that axis is the 2.3 fold excess of the MTs that grow from the apical as compared to those that emerge from the basal cell region. However, other factors may well play a role in the drifting of the nuclei in the polarized follicle cells.

Two important observations suggest that nuclear wriggling and positioning are tightly related processes. (1) The ceasing of nuclear wriggling and positioning at the same time during the course of egg primordia development (Fig. 13). (2) Both nuclear wriggling and positioning come to an end upon the elimination of MTs or when preventing their growth. We thus propose, based 
on the causal relationship between nuclear wriggling and positioning, that nuclear wriggling is a hallmark of a nuclear positioning mechanism described here for the first time.

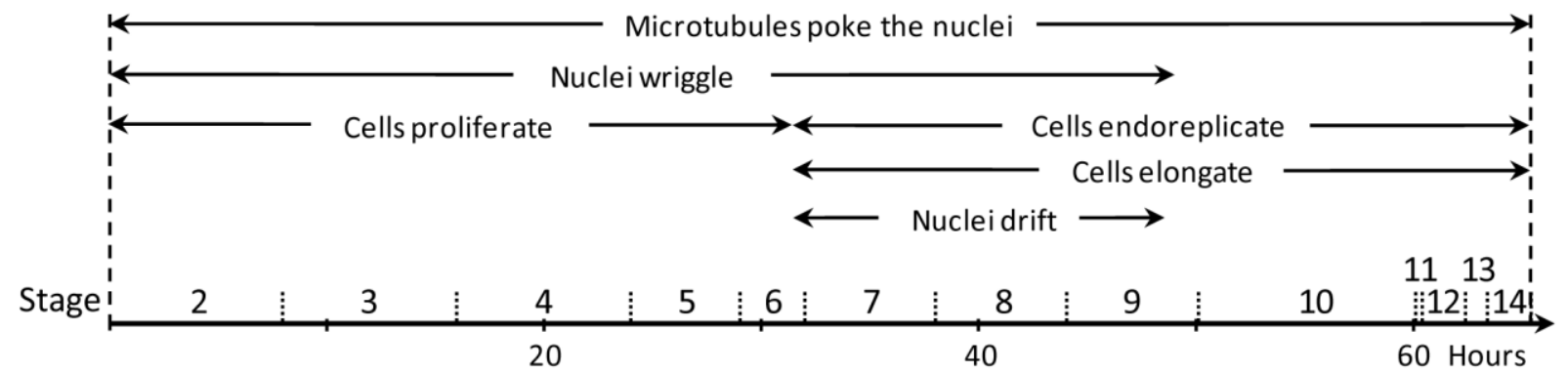

Figure 13. An overview of six processes in the follicle cells during egg primordia development (cf. Ashburner, 2005; Horne-Badovinac and Bilder 2005).

\section{Nuclear Positioning}

In most eukaryotic cells, the nucleus is localized in a specific location (Morris, 2000). However the positions of the nucleus and other organelles are in fact quite dynamic. Nuclei often migrate through the cytoplasm and sometimes far away from the center of the cell. Nuclear positioning is important in the course of a number of processes such as cell division, cell migration and polarization. For example, in a newly fertilized zygote the pronuclei migrate toward each another and into a species-specific location before the first mitosis commences (Fig.1). In the Drosophila embryo, nuclei have to migrate from the central region toward the periphery prior cellularization (Foe et al., 1983) Nuclear positioning is also essential to a variety of polarized cells, such as intestinal brush-border cells and many secreting endocrine cells. Even in single celled organisms, such as budding and fission yeast, tight control exist to position the nucleus correctly during interphase and prior to cell division (Morris, 2000; Tran et al., 2001). In other cases, nuclear migration events reposition nuclei to distant regions of the cell. Examples include living root hairs (Van Bruaene et al., 2001) and developing C. elegans hypodermal cells (Sulston et al., 1983).

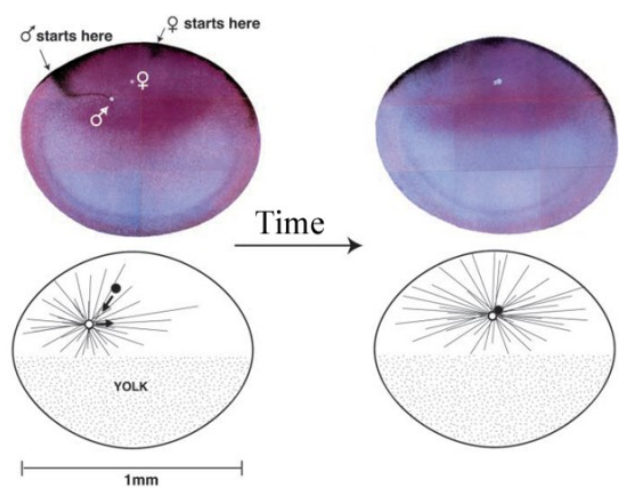

Figure 14. Movements of the female and the male pronuclei following fertilization in a Xenopus egg. The thin black lines are microtubules. The black and white spots are the female and the male pronuclei, respectively. (Histological sections from Hausen and Riebessel 1991). 
The nuclei are targeted to a well-defined site within the cell by one of the so-called nuclear positioning mechanisms (for reviews see Morris, 2003; Starr, 2007; Wilhelmsen, 2006; TolicNørrelykke, 2008 and 2010; Dupin and Etienne-Manneville, 2011). Two related processes are required to control the specific positioning of the nuclei. First, the nuclei must migrate through the cytoplasm to an appropriate position within the cell, and then they must be anchored at that position such that they will not drift away. These processes are controlled by a combination of forces mediated by the cytoskeletal networks. Interactions with the three cytoskeletal filament systems [i.e. F-actin, intermediate filaments and and microtubles] regulate the position of the nucleus and make it move in the cytoplasm. The various filament systems connected to each other through members of the so-called plakin protein family of cytoskeletal cross-linkers (Fuchs and Karakesisoglou, 2001; Leung et al., 2002). Diverse mechanisms exist for migration and anchorage of the nuclei and there are different mechanisms involved in the different cell types and organisms.

\section{The nuclear positioning machineries}

\section{Microfilaments}

The actin cytoskeleton has been known to be involved, directly or indirectly, in nuclear positioning in a number of cell types. In the cleavage Drosophila embryos, for example, nuclei disperse along the anterior-posterior axis in an actin-myosin dependent manner (von Dassow and Schubiger 1994). The force that ensures migration of the nuclei was proposed to originate from depolymerization of an actin gel-like network around the nuclei that actually "surf" in front of the depolymerizing waves (von Dassow and Schubiger 1994; Royou et al., 2002). The actin network has been known to function actively in repositioning the nuclei in the root hairs of Arabidopsis. Drugs that depolymerize the actin cytoskeleton abolish the process, whereas drugs that disrupt the MTs possess no effect (Chytilova et al., 2000). In the budding yeast both the actin filaments and the MTs participate in proper localization of the nuclei and the spindle apparatus to ensure normal cell division (Palmer et al., 1992; Bloom, 2001; Dupin et al. 2011).

\section{Microtubules}

Although in some instances nuclear positioning depends on the actin cytoskeleton, in most studied cases the MTs and the associated dynein and kinesin motors play a central role in 
positioning of nuclei (Fig. 15). Two major types of MT-dependent nuclear positioning mechanisms have been described (reviewed in Reinsch and Gönczy, 1998; Dupin and EtienneManneville, 2011; also see Fig. 15). (1) Most commonly, the nucleus is tightly associated with an MT organizing centre (MTOC) and thus the position of the nucleus follows that of the MTOC. The MTOC can be positioned through (i) the force generated by the emanating astral MTs which - while polymerizing - push against the cell cortex or by (ii) the cortically anchored dynein molecules that pull the decomposing MTs and thus the MTOC-associated nucleus (reviewed in Tolic-Nørrelykke, 2008). (2) Alternatively, the nucleus is transported along the MTs as a cargo to its final destination (Figure 15; Kimura and Onami, 2005).

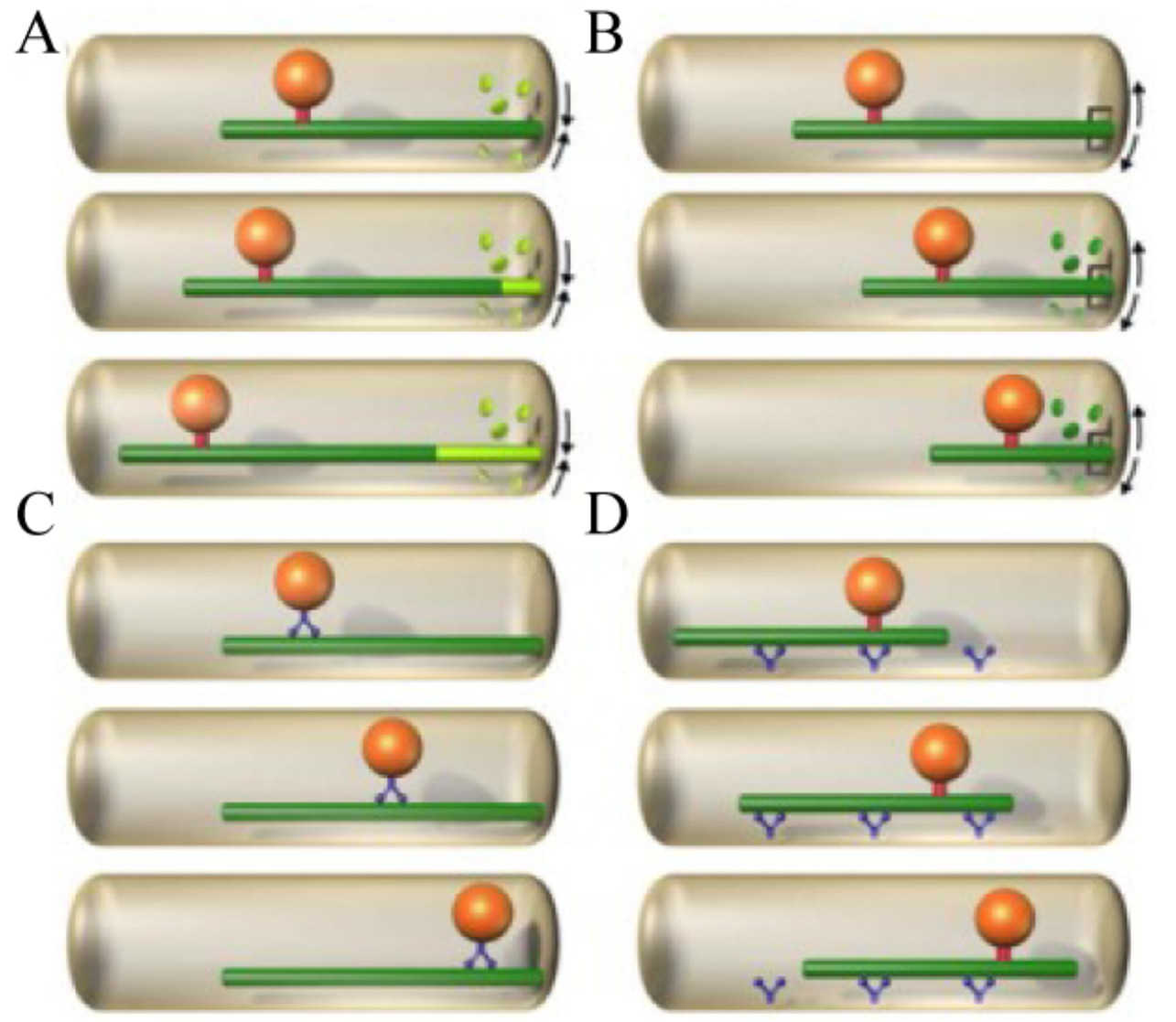

Figure 15. Basic types of microtubule dependent organelle positioning. (A) Pushing, (B) pulling; (C, D) sliding. (A, B) The organelle (orange) is attached to the microtubule (green) by a linker (red). (A) The organelle is being pushed away from the cell edge by the growing MTs. The microtubule polymerizes by addition of new subunits (light green discs) at its $(+)$ end (arrows). (B) A depolymerizing microtubule - connected to the cell edge - pulls the organelle toward the cell edge. Depolymerization is accompanied by a loss of old subunits (dark green discs and arrows). (C) A motor protein (blue) carries the organelle along an MT. (D) Motor proteins (blue) are anchored at the cell cortex and translocate the microtubule together with the bound organelle. Source: Iva M. Tolić-Nørrelykke, 2008. 


\section{Molecular motors}

The forces needed for the correct positioning of the nuclei can be generated by molecular motors and/or polymer motors of the cytoskeleton. Kinesins and dyneins, well known mechanochemical enzymes that are capable to transform chemical energy into movementsa, have been known to be involved in intracellular motions. In almost every reported instance dynein molecules are involved - either directly or indirectly - in MT-dependent nuclear positioning. The cytoplasmic dynein appears to be involved in nuclear positioning by two mechanisms. (1A) The centrosome, that is tightly associated with the nucleus through a dyneindependent manner, nucleates astral MTs which while grow and push against the cell membrane generate the force required to position the nucleus (reviewed in Reinsch and Gönczy, 1998; Gönczy et al., 1999; Morris, 2003; Tolić-Nørrelykke, 2008 and 2010). The process is reminiscent of the growing interpolar MTs that separate the daughter centrosomes (Venkei et al. 2006). Inhibition of dynein activity not only eliminates this type of nuclear positioning but has been shown to bring about an increase in the nucleus-centrosome distance in the neurons (Shu et al., 2004; Tanaka et al., 2004; Tsai, 2005). (1B) In a number of higher eukaryotic organisms dyneins anchored to the cell cortex pull on the on the astral MTs and move the nucleus (reviewed in Dujardin and Vallee, 2002; Alan, 1996; Reinsch and Karsenti 1997). (2) In some instances the dynein motors are linked to the nuclear envelope and while they move along the MTs transport the nucleus to its destination (Rouviere et al., 1994; Schatten, 1994; Allan, 1996; Reinsch and Karsenti, 1997). For example, the Xenopus female pronucleus migrates as a result of dynein on the nuclear envelope pulling the pronucleus towards the male centrosome (Fig. 14). Similarly, studies on pronuclear migration in Caenorhabditis elegans zygotes have also indicated a role for orthologs of dynein heavy chain and Lis1 in nucleokinesis. The Sun1 nuclear envelope protein and the Zyg-12 protein were proposed to mediate the attachment of dynein to the nucleus (Malone et al., 2003). In the filamentous Aspergillus nidulans the nuclei migrate over long distances toward the growing tip of the hyphae and the process requires the fungal homologues of human dynein, dynactin and Lis 1 (reviewed in Tsai and Gleeson, 2005).

Kinesins have also been shown to be involved in some tapes of nuclear positioning. An example comes form $S$. cerevisiae where during mating Kar3p, a kinesin motor, is required to bring the two nuclei together prior to their fusion (reviewed by Rose, 1996). In Schizosaccharomyces pombe Kms1 dynein and/or kinesin-1 involved in nuclear migration. 


\section{"Polymer motors"}

Molecular motors have evolved mechanochemical cycles that enable them to move at hundreds of nanometers per second along their filaments and, generate forces in the range of piconewtons $(\mathrm{pN})$. Polymer motors are less known but also play important roles in various forms of intracellular motility. These motors rely on the relatively simpler processes of polymerization and depolymerization of dynamic biopolymers and on the gelation and solation of cytoskeletal gels (Fig. 16). MTs are polymers of $\alpha$ - and $\beta$-tubulin dimers. Tubulin dimers polymerize end to end in protofilaments. Typically 13 protofilaments associate laterally to form the MT. MTs are the most rigid cytoskeletal filaments, which form a stiff structural scaffold that bears compressive forces in living cells. However MTs are also very dynamic. This property is called dynamic instability which is the intrinsic ability of the MTs to rapidly switch between a growing and a shrinking state, events that are termed catastrophes and rescues (Mitchison and Kirschner, 1984). Dynamic instability allows MTs to interact temporarily with cellular components, to search the intracellular space, to disassemble and assemble into different arrangements and to dynamically position cell organelles (Howard 2006). Thermal fluctuations are necessary between the growing filament and the barrier to create transient gaps that allow for the insertion and assembly of the new subunits. After the insertion of a new subunit, the barrier can no longer diffuse back to its original place and thus the assembling filament is capable to push against the applied load. Hence the load is driven by its own and by the MTs thermal fluctuations (reviewed in Mogilner and Oster, 2003; see also Fig. 16). MTs are believed to grow practically straight and behave as homogeneous elastic rods: they bend as the applied force overcomes their flexural rigidity. Bending or buckling is a clear sign of force generation by the growing MT. Growth often persists after the initiation of the buckling. Force reduces the catastrophe time (the average time the MTs spent in a growing phase until a catastrophe) and the MT/barrier contact time only by limiting the addition of new subunit (Janson et al., 2003). The force can thus be considered constant during buckling. The more force decreases growth velocity the shorter the MT/barrier contact time becomes. The force that a single MT can generate was measured by attaching MTs to a substrate at one end and causing them to push against a microfabricated rigid barrier at the other end. Eexperiments showed that MTs can resist forces of at least $5 \mathrm{pN}$ (Dogterom and Yurke, 1997). These forces may even be higher in vivo. Inoue and Salmon calculated that the maximum pushing force generated by MTs is around $32 \mathrm{pNs}$ in the cells (Inoue and Salmon, 1995). 
A single filament Brownian ratchet
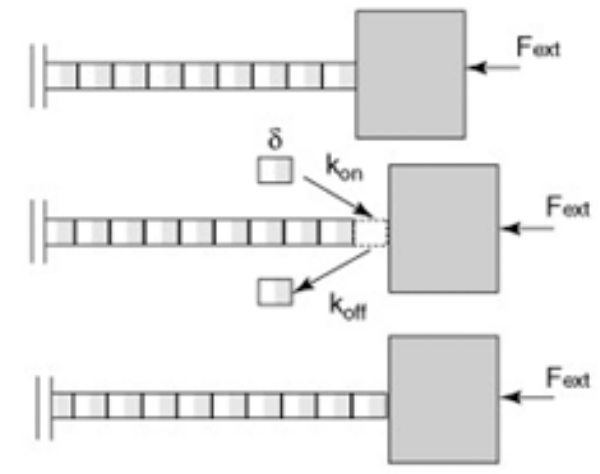

BMultifilament ratchet model
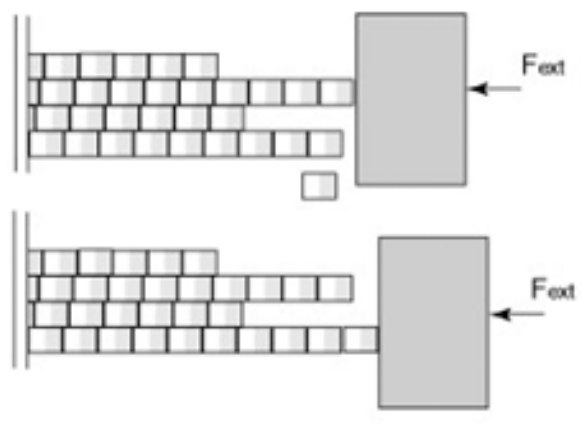

$\mathrm{C}_{\text {Real MT }}$

Growing: pushing

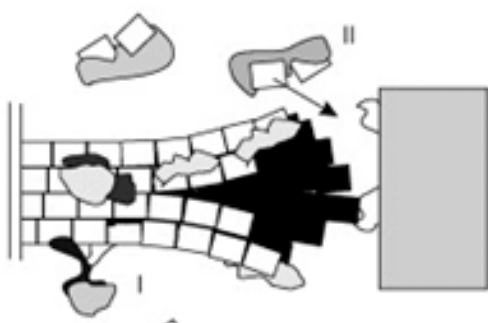

Shrinking: pulling

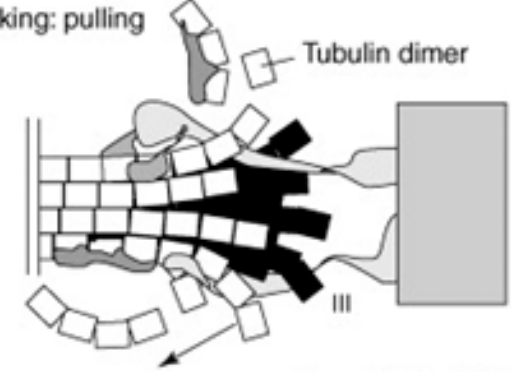

Figure 16. Force generation by MT polarization and depolimerization. (A) Principle of the Brownian ratchet model: thermal fluctuations of the filament and the barrier allow the insertions of new subunits into the polymer. After the insertion of a new subunit the barrier can no longer diffuse back. (B) Generalization of the Brawnian ratchet model for multifilament polymers such as the MTs. (C) Representation of real growing and shrinking MT ends. In the cell, the MT ends are associated with proteins which regulate polymerization, force generation and intracellular interactions. Source: Dogterom et al., 2005.

\section{Anchorage and the LINC complex}

Once in the right position, the nuclei are anchored by the cytoskeleton components (MTs, actin filaments and intermediate filaments), which act either independently or co-operatively to stabilize the position of the nucleus (Wilhelmsen, 2006; Dupin and Etienne-Manneville 2011). LINC (linker of nucleoskeleton and cytoskeleton complexes) connects the nucleus to the cytoskeleton. The nucleoskeleton, which provides structure to the nucleus, is made of lamins, the inner nuclear membrane proteins and the chromosomes. It is separated from the 
cytoskeleton by the nuclear envelope. KASH and SUN proteins form the bridge across the nuclear envelope and interact with the cytoskeleton to move the nuclei to a location and to anchor them (Fig. 17). The cytoplasmic domains of the KASH proteins interact with a variety of components of the cytoskeleton. SUN proteins, in turn, interact with the nucleoskeleton. The entire chain of proteins - from cytoskeletal elements through KASH and SUN bridges to the nucleoskeleton is referred to as the LINC complex. Forces generated by the cytoskeleton and transferred through KASH-SUN bridges are also used to move structures within the nucleus, including meiotic chromosomes. The nucleus responds to mechanical stimuli that are generated outside of the cell and are transmitted to the cell through the cytoskeleton. The nucleus also regulates the organization of the cytoskeleton in a global sense. The KASH proteins (Klarsicht, ANC-1, Syne homology) are C-tail-anchored membrane proteins, which are targeted specifically to the outer membrane of the nuclear envelope (Fig. 17). The large cytoplasmic domains of KASH proteins interact with a variety of cytoskeletal elements. The mammalian Syne/Nesprin-1 and -2, the C. elegans ANC-1, and the Drosophila MSP-300 orthologs are giant proteins with remarkable similarity to dystrophin. They function to connect the nucleus to the actin cytoskeleton in order to anchor or move nuclei (Fig. 17). The Drosophila Klarsicht, the C. elegans UNC-83 and ZYG-12, the mammalian Nesprin-4 and the Schizosaccharomyces pombe Kms1 act as cargo adaptors for the microtubule motors dynein and/or kinesin-1 during nuclear migration. C. elegans ZYG-12 attaches the centrosome to the nuclear envelope. Human Nesprin-3 connects intermediate filaments to nuclei, although the functional significance of this remains unknown. Csm4 in Saccharomyces cerevisiae is thought to couple actin dynamics to the nucleus (Fig. 17). The defining feature of the KASH proteins resides is the carboxyterminal KASH domain that consists of a hydrophobic region spanning the outer nuclear envelope and 6-30 residues in the perinuclear space. The KASH proteins also have large, nonconserved cytoplasmic domains. The SUN proteins (for Sad1 and UNC-84) are integral components of the inner nuclear envelope with conserved, carboxy-terminal SUN domains that localize to the perinuclear space. The SUN domains are conserved across all eukaryotes. Although the nucleoplasmic domains of the SUN proteins are not conserved yet they interact with structural components of the nucleoskeleton; many interact directly with lamins. Importance of a solid anchorage is revealed during the later stages of Drosophila oogenesis. The nurse cells 'dump' their cytoplasmic contents into the oocyte through cytoplasmic bridges (ring canals) and then undergo apoptosis (Spradling, 1993). The positions and the anchorage of the nuclei are thought to be crucial during this process because if they were to become detached they would block the canals (Guild et al., 1997). 


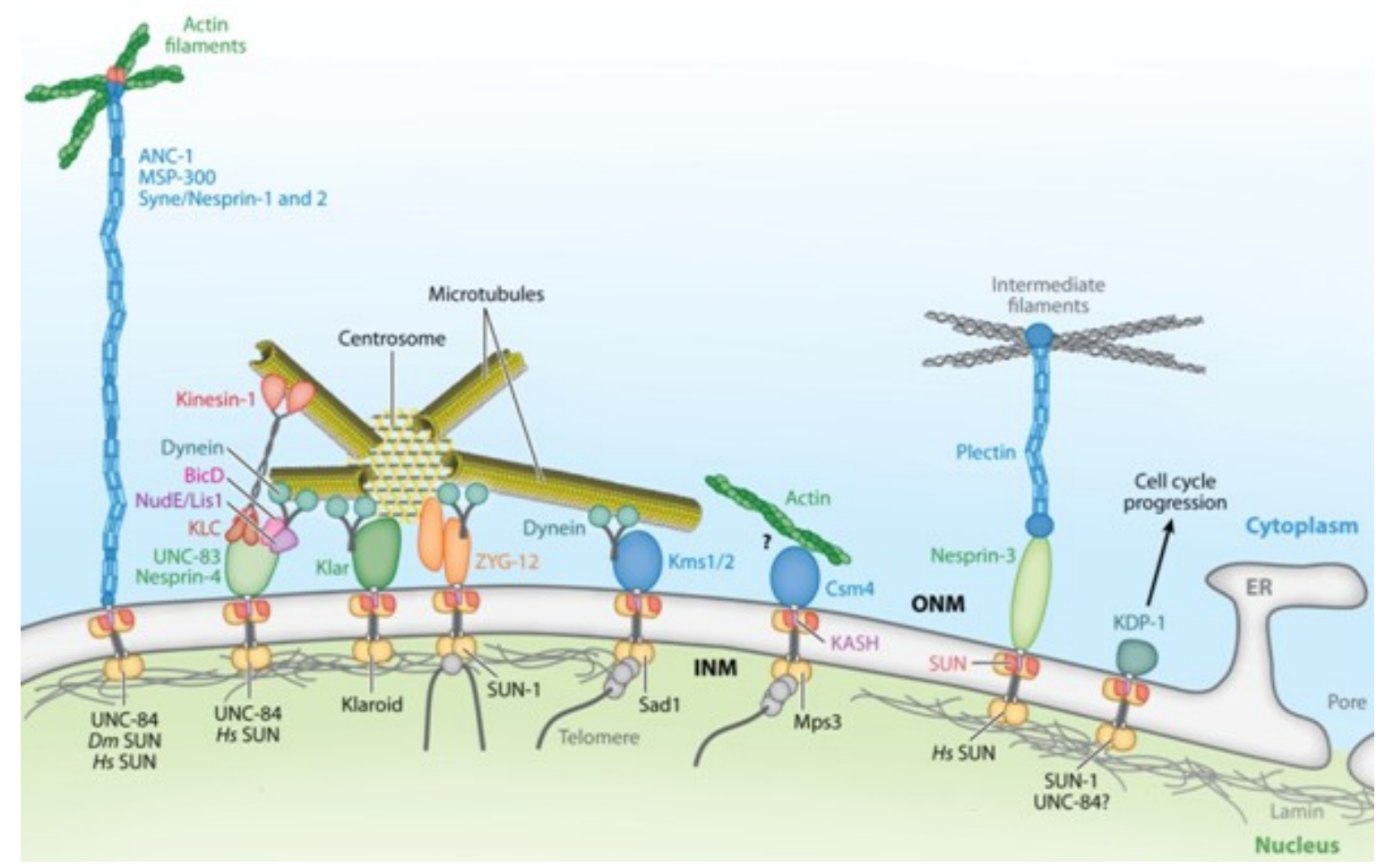

Figure 17. Proteins involved in nuclear positioning and anchorage. KASH proteins are in the outer nuclear membrane (ONM). SUN protein dimers (gold circles) are located in the inner nuclear membrane (INM) with their SUN domains ( red) in the perinuclear space, where they interact with KASH domains (purple) to bridge the nuclear envelope. Source: Starr and Fridolfsson, 2010.

\section{Defective nuclear positioning and diseases}

Defects in the nuclear envelope lead to a wide variety of diseases termed laminopathies.

Several of these diseases are the outcomes of mutations in the genes encoding the SUN or the KASH proteins. These mutations lead to nuclear positioning defects. Distinct perturbations of the nuclear movement and/or anchorage also lead to diseases. The Syne/Nesprin-1 knockout mice phenocopy Emery Dreifuss muscular dystrophy and a subset of the patients with this disease have mutations in the Syne/Nesprin-1 or -2 genes. Mutations in the Syne/Nesprin-1 gene cause autosomal cerebellar ataxia type 1 (ARCA1) that is also known as recessive ataxia of Beauce, a late-onset ataxia with slow progression. Further studies will be required to understand how nuclear positioning contributes to this neurodegenerative disease or if the ataxia represents a defect independendent of the failed KASH function. It also remains to be seen if other human KASH or SUN proteins are also involved in the pathology of these types diseases. Low levels of the KASH proteins have been known to be associazed with progression of the premature aging disease Hutchinson-Gilford progeria syndrome (Fig. 18). 


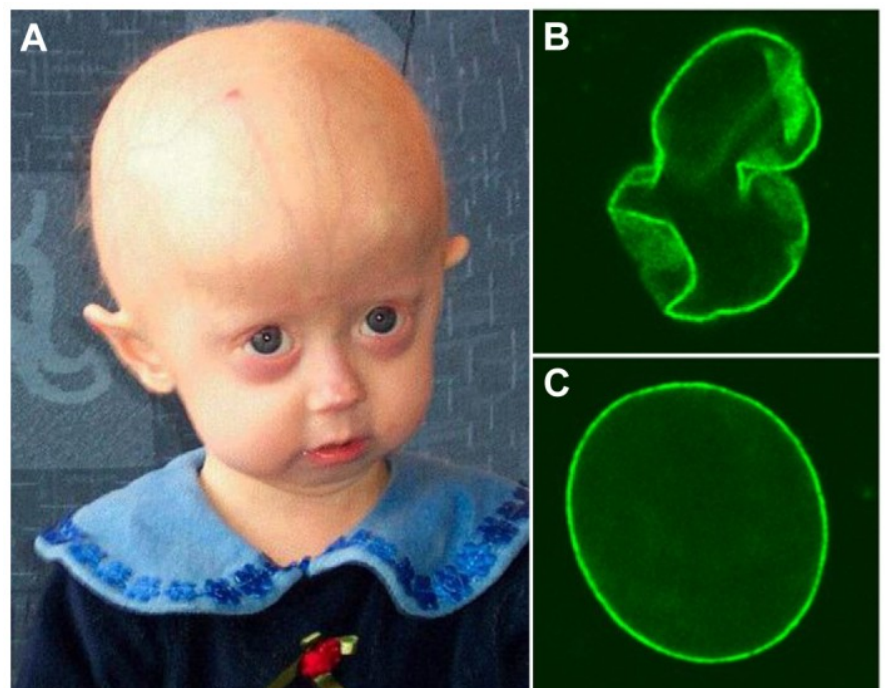

Figure 18. Progeria (also known as "Hutchinson-Gilford Progeria Syndrome) is a rare genetic disease. Symptoms are manifested at an early age and resembling aspects of aging (A). Progeria is one of the so-called laminopathies or nuclear envelopathies. Nuclear envelopaties often caused by affected nucleoskeleton-cytoskeleton interactions. In progeria patients the cell nucleus has dramatically aberrant morphology (B) rather than the uniform shape typically found in healthy individuals (C). Source: http://dx.doi.org/10.1371/journal.pbio.0030395.

Syne/Nesprin-1 has also been linked to Meckel-Gruber syndrome characterized by defective ciliogenesis. Finally, mutations in the Syne/Nesprin-1 and -2 genes have been linked to increased risk for a number of cancers. Disturbance of nuclear migration of the developing cerebral cortex lead to the human disease lissencephaly (Lambert de Rouvroit and Goffinet, 2001; Starr, 2007). Lissencephaly (Fig. 19) (Greek for smooth brain) is a neuro-developmental disease where neurons fail to properly migrate to the cortex. Interkinetic nuclear migration is a common feature of the developing neuroepithelia. Mutations in Doubelcortin, LIS1, dynein and its Aspergillus orthologue NudF cause defective nuclear movement and lead to neuronal migration defects. Neuronal migration in siRNA-treated living and knockout mice clearly showed the role of nuclear migration in the so-called 'lissencephaly mutations'. 


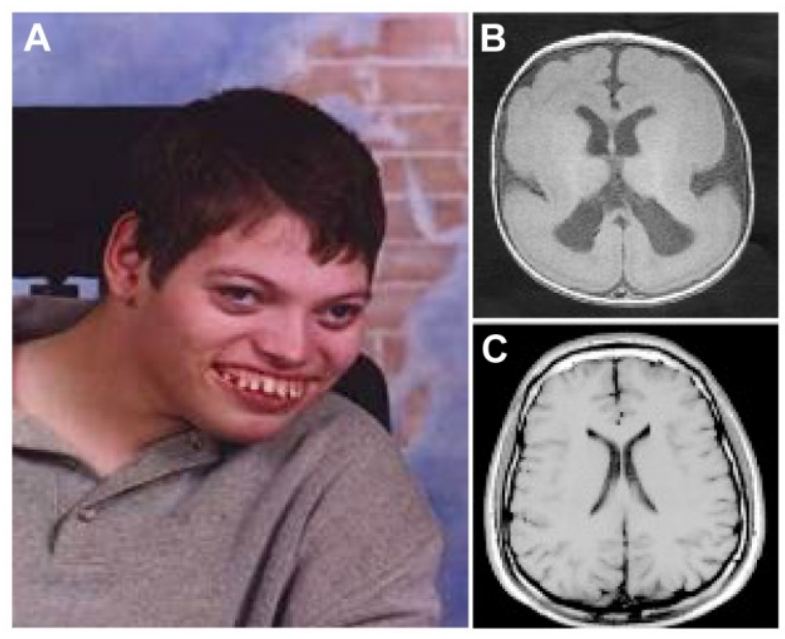

Figure 19. Lissencephaly is a rare brain formation disorder caused by defective neuronal migration. (A) Children with lissencephaly are severely neurologically impaired: they display muscle spasticity or hypotonia. Other symptoms of the disorder may include unusual facial appearance, difficulty swallowing, and anomalies of the hands. Affected children often die within several months of birth. The disorder produce a smooth brain (B) because of lack of migration of immature neurons, without the normal folds of the brain surface $(\mathrm{C})$. Source: webspace.webring.com/people/d1/lfurlotte99/.

In the multinucleate myofibre, individual nuclei are regularly positioned. Improper positioning is a characteristic in many muscle disease including centronuclear myopathies (Jungbluth et al., 2008). Patients show muscle weakness with mislocalized myonuclei. Along with the former observations, the Drosophila Ens (Ensconsin) mutants also possess defective nuclear positioning and decreased larval motility (Metzger et al., 2012). Depleting the Ens orthologues in cultured primary mouse myoblasts and C2C12 myoblasts bring about similar defects. Later in Drosophila development, nuclear migration in the developing Drosophila eye is crutial for the shaping of each individual cell and thus for normal morphology of the compound eye (Fischer-Vize and Mosley, 1994). Signaling of the oocyte nucleus during its migration defines the $\mathrm{A} / \mathrm{P}$ and the $\mathrm{D} / \mathrm{V}$ axes of the oocyte and also determines the polarity of the embryo (van Eeden and St. Johnston, 1999). There is no doubt that future studies on the architecture and positioning of the nucleus and cellular mechanotransduction will reveal many new and exciting functions for this family of proteins. 


\section{ACKNOWLEDGEMENTS}

I wish to thank my scientific supervisor Professor János Szabad for his encouragement, guidance and support throughout my Ph.D. studies. Working on the "Wriggling nuclei" project was a delightful period in my life. Naturally, published results are the results of joint efforts that constitute a coherent story. I am grateful for joint and collaborative work to Imre Gáspár and János Szabad. Let me express my deep gratitude to Imre Gáspár, Zoltán Villányi, Tamás Szalontai, Zsolt Venkei and Farkas Kerényi whom I interacted with and shared pleasures throughout my stay in the Szabad laboratory. I am grateful for the excellent technical support of Gabriella Teleki and Kisné Ani. They all made the Szabad laboratory a loving family. We also thank the Bloomington Drosophila Stock Center for the Drosophila strains, Stefan Heidmann for histone-RFP, Tadashi Uemura for EB1-GFP, Damian Brunner for EB1-Cherry and Nico Stuurman for lamin-GFP. The project was supported by OTKA NI-69180 from the Hungarian Scientific Research Fund and the Graduate Student Program of the University of Szeged. 


\section{REFERENCES}

Allan, V. (1996). Motor proteins: A dynamic duo. Curr. Biol. 6, 630-633.

Ashburner, M., Golic, K.G., Hawley, R.S. (2005). Drosophila: a laboratory handbook, pp 1-1408. New York: Cold Spring Harbor Laboratory Press

Bacallao, R., Antony, C., Dotti, C., Karsenti, E., Stelzer, E. H. and Simons, K. (1989). The subcellular organization of madin-darby canine kidney cells during the formation of a polarized epithelium. J. Cell Biol. 109, 2817-2832.

Bard, F., Bourgeois, C. A., Costagliola, D. and Bouteille, M. (1985). Rotation of the cell nucleus in living cells: A quantitative analysis. Biol. Cell. 54, 135-142.

Bloom, K. (2001). Nuclear migration: Cortical anchors for cytoplasmic dynein. Curr. Biol. 11, R3269.

Bobinnec, Y., Marcaillou, C., Morin, X. and Debec, A. (2003). Dynamics of the endoplasmic reticulum during early development of drosophila melanogaster. Cell Motil. Cytoskeleton 54, 217225.

Bola, B. and Allan, V. (2009). How and why does the endoplasmic reticulum move? Biochem. Soc. Trans. 37, 961-965.

Bouchard, P., Penningroth, S. M., Cheung, A., Gagnon, C. and Bardin, C. W. (1981). Erythro9-[3-(2-hydroxynonyl)]adenine is an inhibitor of sperm motility that blocks dynein ATPase and protein carboxylmethylase activities. Proc. Natl. Acad. Sci. U. S. A. 78, 1033-1036.

Brand, A. H. and Perrimon, N. (1993). Targeted gene expression as a means of altering cell fates and generating dominant phenotypes. Development 118, 401-415.

Brangwynne, C. P., MacKintosh, F. C. and Weitz, D. A. (2007). Force fluctuations and polymerization dynamics of intracellular microtubules. Proc. Natl. Acad. Sci. U. S. A. 104, 1612816133. 
Brouhard, G. J. and Hunt, A. J. (2005). Microtubule movements on the arms of mitotic chromosomes: Polar ejection forces quantified in vitro. Proc. Natl. Acad. Sci. U. S. A. 102, 1390313908.

Burkhardt, J. K., Echeverri, C. J., Nilsson, T. and Vallee, R. B. (1997). Overexpression of the dynamitin ( $p 50$ ) subunit of the dynactin complex disrupts dynein-dependent maintenance of membrane organelle distribution. J. Cell Biol. 139, 469-484.

Buszczak, M., Paterno, S., Lighthouse, D., Bachman, J., Planck, J., Owen, S., Skora, A. D., Nystul, T. G., Ohlstein, B., Allen, A. et al. (2007). The carnegie protein trap library: A versatile tool for drosophila developmental studies. Genetics 175, 1505-1531.

Chytilova, E., Macas, J., Sliwinska, E., Rafelski, S. M., Lambert, G. M. and Galbraith, D. W. (2000). Nuclear dynamics in arabidopsis thaliana. Mol. Biol. Cell 11, 2733-2741.

Clark, I. E., Jan, L. Y. and Jan, Y. N. (1997). Reciprocal localization of nod and kinesin fusion proteins indicates microtubule polarity in the drosophila oocyte, epithelium, neuron and muscle. Development 124, 461-470.

De Boni, U. and Mintz, A. H. (1986). Curvilinear, three-dimensional motion of chromatin domains and nucleoli in neuronal interphase nuclei. Science 234, 863-866.

Dej, K. J. and Spradling, A. C. (1999). The endocycle controls nurse cell polytene chromosome structure during drosophila oogenesis. Development 126, 293-303.

Delmar, V. A., Chan, R. C. and Forbes, D. J. (2008). Xenopus importin beta validates human importin beta as a cell cycle negative regulator. BMC Cell Biol. 9, 14.

Dogterom, M., Kerssemakers, J. W., Romet-Lemonne, G. and Janson, M. E. (2005). Force generation by dynamic microtubules. Curr. Opin. Cell Biol. 17, 67-74.

Dogterom, M. and Yurke, B. (1997). Measurement of the force-velocity relation for growing microtubules. Science 278, 856-860.

Duffy, J. B. (2002). GAL4 system in drosophila: A fly geneticist's swiss army knife. Genesis 34, 115. 
Dujardin, D. L. and Vallee, R. B. (2002). Dynein at the cortex. Curr. Opin. Cell Biol. 14, 44-49.

Dupin, I. and Etienne-Manneville, S. (2011). Nuclear positioning: Mechanisms and functions. Int.

J. Biochem. Cell Biol. 43, 1698-1707.

Dupin, I., Sakamoto, Y. and Etienne-Manneville, S. (2011). Cytoplasmic intermediate filaments mediate actin-driven positioning of the nucleus. J. Cell. Sci. 124, 865-872.

Echeverri, C. J., Paschal, B. M., Vaughan, K. T. and Vallee, R. B. (1996). Molecular characterization of the 50-kD subunit of dynactin reveals function for the complex in chromosome alignment and spindle organization during mitosis. J. Cell Biol. 132, 617-633.

Elbaum, M., Kuchnir Fygenson, D. and Libchaber, A. (1996). Buckling microtubules in vesicles. Phys. Rev. Lett. 76, 4078-4081.

Felgner, H., Frank, R., Biernat, J., Mandelkow, E. M., Mandelkow, E., Ludin, B., Matus, A. and Schliwa, M. (1997). Domains of neuronal microtubule-associated proteins and flexural rigidity of microtubules. J. Cell Biol. 138, 1067-1075.

Fischer-Vize, J. A. and Mosley, K. L. (1994). Marbles mutants: Uncoupling cell determination and nuclear migration in the developing drosophila eye. Development 120, 2609-2618.

Foe, V. E. and Alberts, B. M. (1983). Studies of nuclear and cytoplasmic behaviour during the five mitotic cycles that precede gastrulation in drosophila embryogenesis. J. Cell. Sci. 61, 31-70.

Frescas, D., Mavrakis, M., Lorenz, H., Delotto, R. and Lippincott-Schwartz, J. (2006). The secretory membrane system in the drosophila syncytial blastoderm embryo exists as functionally compartmentalized units around individual nuclei. J. Cell Biol. 173, 219-230.

Fuchs, E. and Karakesisoglou, I. (2001). Bridging cytoskeletal intersections. Genes Dev. 15, 114.

Gaspar, I. and Szabad, J. (2009). Glu415 in the alpha-tubulins plays a key role in stabilizing the microtubule-ADP-kinesin complexes. J. Cell. Sci. 122, 2857-2865. 
Gaspar, I. and Szabad, J. (2009). In vivo analysis of MT-based vesicle transport by confocal reflection microscopy. Cell Motil. Cytoskeleton 66, 68-79.

Goldmann, W. H. (2002). Examination of actin polymerization and viscosity induced by cations and ionic strength when cross-linked by alpha-actinin. Cell Biol. Int. 26, 541-546.

Gonczy, P., Pichler, S., Kirkham, M. and Hyman, A. A. (1999). Cytoplasmic dynein is required for distinct aspects of MTOC positioning, including centrosome separation, in the one cell stage caenorhabditis elegans embryo. J. Cell Biol. 147, 135-150.

Guild, G. M., Connelly, P. S., Shaw, M. K. and Tilney, L. G. (1997). Actin filament cables in drosophila nurse cells are composed of modules that slide passively past one another during dumping. J. Cell Biol. 138, 783-797.

Hampoelz, B., Azou-Gros, Y., Fabre, R., Markova, O., Puech, P. H. and Lecuit, T. (2011). Microtubule-induced nuclear envelope fluctuations control chromatin dynamics in drosophila embryos. Development 138, 3377-3386.

Hausen, P. and Riebessell, M. (1991). The early development of Xenopus laevis. pp. 142. Springer-Verlag, Berlin.

Herbomel, P. (1999). Spinning nuclei in the brain of the zebrafish embryo. Curr. Biol. 9, R627-8.

Horne-Badovinac, S. and Bilder, D. (2005). Mass transit: Epithelial morphogenesis in the drosophila egg chamber. Dev. Dyn. 232, 559-574.

Howard, J. (2006). Elastic and damping forces generated by confined arrays of dynamic microtubules. Phys. Biol. 3, 54-66.

Inoue, S. and Salmon, E. D. (1995). Force generation by microtubule assembly/disassembly in mitosis and related movements. Mol. Biol. Cell 6, 1619-1640.

Janson, M. E., de Dood, M. E. and Dogterom, M. (2003). Dynamic instability of microtubules is regulated by force. J. Cell Biol. 161, 1029-1034. 
Jungbluth, H., Wallgren-Pettersson, C. and Laporte, J. (2008). Centronuclear (myotubular) myopathy. Orphanet J. Rare Dis. 3, 26.

Karpova, N., Bobinnec, Y., Fouix, S., Huitorel, P. and Debec, A. (2006). Jupiter, a new drosophila protein associated with microtubules. Cell Motil. Cytoskeleton 63, 301-312.

Kimura, A. and Onami, S. (2005). Computer simulations and image processing reveal lengthdependent pulling force as the primary mechanism for C. elegans male pronuclear migration. Dev. Cell. 8, 765-775.

King, R. C. (1970). Ovarian development in Drosophila melanogaster, pp 1-227. New York: Academic Press.

Laan, L. and Dogterom, M. (2010). In vitro assays to study force generation at dynamic microtubule ends. Methods Cell Biol. 95, 617-639.

Lambert de Rouvroit, C. and Goffinet, A. M. (2001). Neuronal migration. Mech. Dev. 105, 47-56.

Lau, C. K., Delmar, V. A., Chan, R. C., Phung, Q., Bernis, C., Fichtman, B., Rasala, B. A. and Forbes, D. J. (2009). Transportin regulates major mitotic assembly events: From spindle to nuclear pore assembly. Mol. Biol. Cell 20, 4043-4058.

Leung, C. L., Green, K. J. and Liem, R. K. (2002). Plakins: A family of versatile cytolinker proteins. Trends Cell Biol. 12, 37-45.

Li, T. (2008). A mechanics model of microtubule buckling in living cells. J. Biomech. 41, 1722-1729.

Lother, H., Blitstein-Willinger, E. and Diamantstein, T. (1979). Studies on the relevance of microtubules and of microfilament-dependent processes for triggering lymphocyte activation. $Z$. Immunitatsforsch. Immunobiol. 155, 346-358.

Malone, C. J., Misner, L., Le Bot, N., Tsai, M. C., Campbell, J. M., Ahringer, J. and White, J. G. (2003). The C. elegans hook protein, ZYG-12, mediates the essential attachment between the centrosome and nucleus. Cell 115, 825-836. 
Metzger, T., Gache, V., Xu, M., Cadot, B., Folker, E. S., Richardson, B. E., Gomes, E. R. and Baylies, M. K. (2012). MAP and kinesin-dependent nuclear positioning is required for skeletal muscle function. Nature 484, 120-124.

Mickey, B. and Howard, J. (1995). Rigidity of microtubules is increased by stabilizing agents. J. Cell Biol. 130, 909-917.

Mitchison, T. and Kirschner, M. (1984). Dynamic instability of microtubule growth. Nature 312, 237-242.

Miyamoto, Y., Muto, E., Mashimo, T., Iwane, A. H., Yoshiya, I. and Yanagida, T. (2000). Direct inhibition of microtubule-based kinesin motility by local anesthetics. Biophys. J. 78, 940-949.

Mogilner, A. and Oster, G. (2003). Polymer motors: Pushing out the front and pulling up the back. Curr. Biol. 13, R721-33.

Morris, N. R. (2000). Nuclear migration. from fungi to the mammalian brain. J. Cell Biol. 148, 1097-1101.

Morris, N. R. (2003). Nuclear positioning: The means is at the ends. Curr. Opin. Cell Biol. 15, 5459.

Paddock, S. W. and Albrecht-Buehler, G. (1986). Distribution of microfilament bundles during rotation of the nucleus in 3 T3 cells treated with monensin. Exp. Cell Res. 163, 525-538.

Paddock, S. W. and Albrecht-Buehler, G. (1988). Rigidity of the nucleus during nuclear rotation in $3 T 3$ cells. Exp. Cell Res. 175, 409-413.

Palmer, R. E., Sullivan, D. S., Huffaker, T. and Koshland, D. (1992). Role of astral microtubules and actin in spindle orientation and migration in the budding yeast, saccharomyces cerevisiae. J. Cell Biol. 119, 583-593. 
Park, E. A., Macalpine, D. M. and Orr-Weaver, T. L. (2007). Drosophila follicle cell amplicons as models for metazoan DNA replication: A cyclinE mutant exhibits increased replication fork elongation. Proc. Natl. Acad. Sci. U. S. A. 104, 16739-16746.

POMERAT, C. M. (1953). Rotating nuclei in tissue cultures of adult human nasal mucosa. Exp. Cell Res. 5, 191-196.

Reinsch, S. and Gonczy, P. (1998). Mechanisms of nuclear positioning. J. Cell. Sci. 111 ( Pt 16), 2283-2295.

Reinsch, S. and Karsenti, E. (1997). Movement of nuclei along microtubules in xenopus egg extracts. Curr. Biol. 7, 211-214.

Rolls, M. M., Satoh, D., Clyne, P. J., Henner, A. L., Uemura, T. and Doe, C. Q. (2007). Polarity and intracellular compartmentalization of drosophila neurons. Neural Dev. 2, 7.

Rose, M. D. (1996). Nuclear fusion in the yeast saccharomyces cerevisiae. Annu. Rev. Cell Dev. Biol. 12, 663-695.

Rouviere, C., Houliston, E., Carre, D., Chang, P. and Sardet, C. (1994). Characteristics of pronuclear migration in beroe ovata. Cell Motil. Cytoskeleton 29, 301-311.

Royou, A., Sullivan, W. and Karess, R. (2002). Cortical recruitment of nonmuscle myosin II in early syncytial drosophila embryos: Its role in nuclear axial expansion and its regulation by Cdc2 activity. J. Cell Biol. 158, 127-137.

Schatten, G. (1994). The centrosome and its mode of inheritance: The reduction of the centrosome during gametogenesis and its restoration during fertilization. Dev. Biol. 165, 299-335.

Schuh, M., Lehner, C. F. and Heidmann, S. (2007). Incorporation of drosophila CID/CENP-A and CENP-C into centromeres during early embryonic anaphase. Curr. Biol. 17, 237-243.

Shu, T., Ayala, R., Nguyen, M. D., Xie, Z., Gleeson, J. G. and Tsai, L. H. (2004). Ndel1 operates in a common pathway with LIS1 and cytoplasmic dynein to regulate cortical neuronal positioning. Neuron 44, 263-277 
Spradling, A. C. (1993). Germline cysts: Communes that work. Cell 72, 649-651.

Starr, D. A. (2007). Communication between the cytoskeleton and the nuclear envelope to position the nucleus. Mol. Biosyst 3, 583-589.

Starr, D. A. and Fridolfsson, H. N. (2010). Interactions between nuclei and the cytoskeleton are mediated by SUN-KASH nuclear-envelope bridges. Annu. Rev. Cell Dev. Biol. 26, 421-444.

Stewart, M. (2007). Ratcheting mRNA out of the nucleus. Mol. Cell 25, 327-330.

Sulston, J. E., Schierenberg, E., White, J. G. and Thomson, J. N. (1983). The embryonic cell lineage of the nematode caenorhabditis elegans. Dev. Biol. 100, 64-119.

Tanaka, T., Serneo, F. F., Higgins, C., Gambello, M. J., Wynshaw-Boris, A. and Gleeson, J. G. (2004). Lis1 and doublecortin function with dynein to mediate coupling of the nucleus to the centrosome in neuronal migration. J. Cell Biol. 165, 709-721.

Tirian, L., Timinszky, G. and Szabad, J. (2003). P446L-importin-beta inhibits nuclear envelope assembly by sequestering nuclear envelope assembly factors to the microtubules. Eur. J. Cell Biol. 82, 351-359.

Tolic-Norrelykke, I. M. (2010). Force and length regulation in the microtubule cytoskeleton: Lessons from fission yeast. Curr. Opin. Cell Biol. 22, 21-28.

Tolic-Norrelykke, I. M. (2008). Push-me-pull-you: How microtubules organize the cell interior. Eur. Biophys. J. 37, 1271-1278.

Tran, P. T., Marsh, L., Doye, V., Inoue, S. and Chang, F. (2001). A mechanism for nuclear positioning in fission yeast based on microtubule pushing. J. Cell Biol. 153, 397-411.

Tsai, J. W., Chen, Y., Kriegstein, A. R. and Vallee, R. B. (2005). LIS1 RNA interference blocks neural stem cell division, morphogenesis, and motility at multiple stages. J. Cell Biol. 170, 935-945.

Tsai, L. H. and Gleeson, J. G. (2005). Nucleokinesis in neuronal migration. Neuron 46, 383-388. 
Van Bruaene, N., Joss, G. and Van Oostveldt, P. (2001). Computer assisted track analysis of nuclear migration in living root hairs. Meded. Rijksuniv. Gent Fak. Landbouwkd Toegep Biol. Wet. 66, 35-39.

van Eeden, F. and St Johnston, D. (1999). The polarisation of the anterior-posterior and dorsalventral axes during drosophila oogenesis. Curr. Opin. Genet. Dev. 9, 396-404.

Venkei, Z., Gaspar, I., Toth, G. and Szabad, J. (2006). alpha4-tubulin is involved in rapid formation of long microtubules to push apart the daughter centrosomes during earlyx drosophila embryogenesis. J. Cell. Sci. 119, 3238-3248.

Vermaak, D., Hayden, H. S. and Henikoff, S. (2002). Centromere targeting element within the histone fold domain of cid. Mol. Cell. Biol. 22, 7553-7561.

Villanyi, Z., Debec, A., Timinszky, G., Tirian, L. and Szabad, J. (2008). Long persistence of importin-beta explains extended survival of cells and zygotes that lack the encoding gene. Mech. Dev. 125, 196-206.

von Dassow, G. and Schubiger, G. (1994). How an actin network might cause fountain streaming and nuclear migration in the syncytial drosophila embryo. J. Cell Biol. 127, 1637-1653.

Wilhelmsen, K., Ketema, M., Truong, H. and Sonnenberg, A. (2006). KASH-domain proteins in nuclear migration, anchorage and other processes. J. Cell. Sci. 119, 5021-5029.

Yu, J., Starr, D. A., Wu, X., Parkhurst, S. M., Zhuang, Y., Xu, T., Xu, R. and Han, M. (2006). The KASH domain protein MSP-300 plays an essential role in nuclear anchoring during drosophila oogenesis. Dev. Biol. 289, 336-345.

Zhao, T., Graham, O. S., Raposo, A. and St Johnston, D. (2012). Growing microtubules push the oocyte nucleus to polarize the drosophila dorsal-ventral axis. Science. 